\title{
Analysis of an Inverse First Passage Problem from Risk Management
}

\author{
Lan Cheng, Xinfu Chen, John Chadam \& David Saunders ${ }^{\dagger}$
}

January 5, 2005

\begin{abstract}
We study the following "inverse first passage time" problem. Given a diffusion process $X_{t}$ and a probability distribution $q$ on $[0, \infty)$, does there exist a boundary $b(t)$ such that $q(t)=\mathbb{P}[\tau \leq t]$, where $\tau$ is the first hitting time of $X_{t}$ to the time dependent level $b(t)$. A free boundary problem for a parabolic partial differential operator is associated with the inverse first passage time problem. We prove the existence and uniqueness of a viscosity solution to this equation. We also investigate the small time behavior of the boundary $b(t)$, presenting both upper and lower bounds. Finally, we derive some integral equations charaterizing the boundary.
\end{abstract}

\section{Introduction.}

In this paper we study the following free boundary problem: find a boundary $x=b(t)$ $(t>0)$ and an unknown function $w=w(x, t)(x \in \mathbb{R}, t \geq 0)$ such that

$$
\begin{cases}w_{t}(x, t)=\frac{1}{2}\left(\sigma^{2} w_{x}\right)_{x}-\mu w_{x} & \text { for } x>b(t), t>0 \\ w(x, t)=p(t) & \text { for } x \leq b(t), t>0 \\ 0 \leq w(x, t)<p(t) & \text { for } x>b(t), t>0, \\ w(x, 0)=\mathbf{1}_{(-\infty, 0)}(x) & \text { for } x \in \mathbb{R}, t=0,\end{cases}
$$

where $q(t)=1-p(\cdot)$ is a given cumulative probability distribution function with the following properties:

$$
1=p(0)=\lim _{t \searrow 0} p(t), \quad p\left(t_{1}\right) \geq p\left(t_{2}\right) \geq 0 \quad \forall t_{1}<t_{2} .
$$

${ }^{*}$ Xinfu Chen acknowledges support from NSF grant DMS-0203991.

${ }^{\dagger}$ David Saunders acknowledges support from NSF grant DMS-0310656. 
This problem arises from the consideration of the first passage times of diffusion processes to curved boundaries. More specifically, we let $X_{t}$ be the solution of the following stochastic differential equation:

$$
d X_{t}=\mu\left(X_{t}, t\right) d t+\sigma\left(X_{t}, t\right) d B_{t} \quad X_{0}=0
$$

where $B_{t}$ is a standard Brownian motion on a filtered probability space satisfying the usual conditions, and $\mu: \mathbb{R} \times \mathbb{R}_{+} \rightarrow \mathbb{R}$ and $\sigma: \mathbb{R} \times \mathbb{R}_{+} \rightarrow \mathbb{R}$ and smooth bounded functions, and $\sigma(x, t)>\varepsilon>0$ for all $x \in \mathbb{R}, t \geq 0$. For a given function $b: \mathbb{R}_{+} \rightarrow \mathbb{R}$ we define the first passage time of the diffusion process $X_{t}$ to the curved boundary $b(t)$ to be:

$$
\tau=\inf \left\{t>0 \mid X_{t} \leq b(t)\right\}
$$

Two important problems concerning the first passage time of a diffusion process to a curved boundary are the following:

1. The first passage problem: Given a barrier function $b(t)$, find the survival probability $p(t)$ that $X$ does not cross $b$ before or at $t$.

$$
p(t):=\mathbb{P}\{\tau>t\}
$$

2. The inverse first passage problem: Given a survival probability function $p(t)$, find a barrier function $b(t)$, such that (1.5) holds.

The first passage problem is a classical problem in probability, and is the subject of a rather large literature. It is also fundamental in many applications of diffusion processes to engineering, physics, biology and economics. For a survey of techniques for approximating and computing first passage times to curved boundaries, and a discussion of their applications in the biological sciences, we refer to [14]. For some applications in economics closely related to those that motivated this study, we refer to [2].

The work of Peskir [13], [12] on the first passage problem is of particular relevance for the inverse problem discussed in this paper. In [13], he derived a sequence of integral equations $^{1}$

$$
t^{n / 2} H_{n}\left(\frac{b(t)}{\sqrt{t}}\right)+\int_{0}^{t}(t-s)^{n / 2} H_{n}\left(\frac{b(t)-b(s)}{\sqrt{t-s}}\right) \dot{p}(s) d s=0, n=-1,0,1 \ldots
$$

\footnotetext{
${ }^{1}$ In this reference, the derivations are carried out for the case $\sigma \equiv 1$ and $\mu \equiv 0$, i.e. when $X_{t}$ is a Brownian motion. As mentioned in the reference, the techniques directly extend to other diffusion processes.
} 
where $H_{-1}(x)=\frac{1}{\sqrt{2 \pi}} e^{-x^{2} / 2}$ and $H_{n}(x)=\int_{x}^{\infty} H_{n-1}(z) d z$ for $n \geq 0$. In [12], under the assumption that $b(t)$ is $C^{1}$ on $(0, \infty)$, decreasing, and concave, he derived the equality

$$
\dot{p}(0+)=-\lim _{t \searrow 0} \frac{1}{2 \sqrt{2 \pi}} \frac{b(t)}{t^{3 / 2}} e^{-\frac{b^{2}(t)}{2 t}}=-\lim _{t \searrow 0} \frac{\dot{b}(t)}{\sqrt{2 \pi t}} e^{-\frac{b^{2}(t)}{2 t}},
$$

provided that the second or third limit exists.

The inverse first passage problem is much harder than the direct problem and there are only a few studies about it. These are principally concerned with the numerical calculation of the boundary $b(t)$ for a given $p(t)$. There is no publication proving the well-posedness (existence and uniqueness) of the boundary given the survival probability.

Our interest in the inverse first passage problem originates ${ }^{2}$ from Merton's structural model [11] for credit risk management. Consider a company whose asset value and debt at time $t \geq 0$ are denoted by $A_{t}$ and $D_{t}$ respectively. Assume the following:

1. $D_{0} \leq A_{0}$ and the company is in default at a time $t>0$ if $A_{t}<D_{t}$.

2. $A_{t}$ follows a geometric Brownian motion.

It is convenient to use the default index $X_{t}$ and the barrier function $b(t)$ defined by

$$
X_{t}:=\log \frac{A_{t}}{A_{0}}, \quad b(t):=\log \frac{D_{t}}{A_{0}} .
$$

Then $X_{t}$ is a diffusion process satisfying (1.3). In this context, the inverse first passage time problem is the problem of finding the default barrier $b(t)$ given the survival function $p(t)$ (which may, for example, be inferred from the credit spreads on bonds).

Formulating the problems in a PDE setting, we introduce a new function $w(x, t)$ being the probability that the company does not default before or at $t$ and its default index $X_{t}$ is bigger than $x$, i.e.,

$$
w(x, t):=\mathbb{P}\left\{X_{t}>x, \tau>t\right\} .
$$

Then the density function of $X_{t}$ when $\tau>t$ can be computed by

$$
u(x, t)=\frac{d}{d x} \mathbb{P}\{X(t) \leq x, \tau>t\}=(p(t)-w(x, t))_{x} .
$$

From (1.3) and the Kolmogorov forward equation, we see that (assuming sufficient regularity) $w(x, t)(x \in \mathbb{R}, t \geq 0)$ satisfies (1.1). From this we see the following:

\footnotetext{
${ }^{2}$ We thank A. Kreinin and R. Stamicar for introducing us to this problem, and for helpful discussions.
} 
- The first passage problem is to solve (1.1) for $p$, with given $b$.

- The inverse first passage problem is to solve (1.1) for $b$, with given $p$.

The first passage problem can be solved as follows. From the Kolmogorov forward equation, we obtain the following closed system for $u(x, t)$

$$
\begin{cases}u_{t}(x, t)=\frac{1}{2}\left(\sigma^{2} u\right)_{x x}-(\mu u)_{x} & \text { for } x>b(t), t>0 \\ u(b(t), t)=0 & \text { for } x \leq b(t), t>0 \\ u(x, 0)=\delta(x) & \text { for } x>0, t=0\end{cases}
$$

where $\delta$ is a Dirac measure concentrated at 0 . Given sufficiently regular $b$, this system has a unique solution. Then $p$ and $\dot{p}$ can be computed from the formulas

$$
\begin{array}{ll}
p(t)=\int_{b(t)}^{\infty} u(x, t) d x & \forall t \geq 0, \\
\dot{p}(t)=-\left.\frac{1}{2}\left(\sigma^{2} u\right)_{x}\right|_{x=b(t)} & \forall t \geq 0 .
\end{array}
$$

It is only possible to compute the solution in a closed form in a few special cases. However, there is a large literature on numerical and analytic approximations of the solution.

Avellaneda and Zhu [1] were the first to use (1.9) and (1.11) to study the inverse first passage problem. They performed a change of variables from $X_{t}$ to $Y_{t}=X_{t}-b(t)$, whose financial meaning is the risk-neutral distance-to-default process (RNDD) for the company. Denote by $f(y, t)=u(y+b(t), t)$ the probability density function of $Y_{t}$ when $\tau>t$. (1.9) and (1.11) are equivalent to:

$$
\begin{cases}f_{t}=\dot{b}(t) f_{y}-(\mu f)_{y}+\frac{1}{2}\left(\sigma^{2} f_{y}\right)_{y} & \text { for } y>0, t>0 \\ f(0, t)=0 & \text { for } y=0, t>0 \\ f(y, 0)=\delta_{0}(y-b(0)) & \text { for } y>0, t=0 \\ \frac{1}{2} \sigma^{2} f_{y}(0, t)+\dot{p}(t)=0 & \text { for } y=0, t>0\end{cases}
$$

In [8], I.Iscoe and A.Kreinin demonstrated that a Monte Carlo approach can be applied to solve the inverse first-passage problem in discrete time, essentially by reducing it to the sequential estimation of conditional distributions. In [15], both a Monte Carlo algorithm based on a piecewise linear approximation of the boundary, and a method based on the integral equation (1.6) with $n=1$ are studied.

In this paper, we are particularly interested in the following fundamental questions: (1) Given a survival probability function $p(t)$ satisfying (1.2), is there a barrier function $b(t)$ ? 
(2) If there is a barrier function, how many are there? Namely, we are concerned about the well-posedness (existence and uniqueness) of the free boundary problem (1.1).

We point out that solutions to (1.1) are not smooth, so that a notion of weak solution has to be used. Instead of using the classical weak solution defined in the distributional sense (see Evans [4]), we use viscosity solutions, introduced by Crandall and Lions in 1981. In this paper, we shall prove the following theorem.

Theorem 1. Problem (1.1) is a well-posed problem, i.e., for any given $p(t)$ satisfying (1.2), there is a unique (weak) solution.

The remainder of the paper is organized as follows. In $\S 2$, we provide a definition of the viscosity solution to (1.1) and show there is at most one such solution. In $\S 3$, we establish the existence of a viscosity solution. First we define and study a regularization of the problem obtained by penalizing the obstacle (a standard procedure for the obstacle problem, see Friedman [6]). The $\varepsilon$-regularization is carefully designed so that the solution is monotonic in $\varepsilon$, and therefore the existence of a limit as $\varepsilon \rightarrow 0$ is automatically guaranteed. We show that the limit is a viscosity solution. In $\S 4$, we study the asymptotic behavior of the boundary as $t \searrow 0$ by providing explicit upper and lower bounds. When $\lim \sup _{t \searrow 0}-\frac{1-p(t)}{t \dot{p}(t)}<\infty$, we prove that

$$
\lim _{t \rightarrow 0} \frac{b(t)}{\sqrt{-2 t \log (1-p(t))}}=-1 .
$$

In $\S 5$, we derive the integral equations for $b$ when $\sigma \equiv 1$ and $\mu \equiv 0$ under the assumption that $p$ is continuous and non-increasing.

\section{Viscosity Solutions and Uniqueness}

By noticing that $w(x, t)<p(t)$ for all $x>b(t)$ when $\tau>t$, we can state the inverse first passage problem as follows. Find an unknown function $w=w(x, t)$ such that,

$$
\begin{cases}\mathcal{L} w=0 & \text { when } w(\cdot, t)<p(t), \\ 0 \leq w(x, t) \leq p(t) & \text { for any }(x, t) \in(\mathbb{R} \times(0, \infty)), \\ w(x, 0)=\mathbf{1}_{(-\infty, 0)}(x) & \text { for }(x, t) \in(\mathbb{R} \times[0, \infty)),\end{cases}
$$

where $\mathcal{L} w:=w_{t}-\frac{1}{2}\left(\sigma^{2} w_{x}\right)_{x}+\mu w_{x}$. Define the free boundary as:

$$
b^{w}(t):=\inf \{x \mid w(x, t)<p(t)\} .
$$


We can write (2.1) as a variational inequality:

$$
\begin{cases}\max \{\mathcal{L} w, w-p\}=0 & \text { in } \mathbb{R} \times(0, \infty), \\ w(\cdot, 0)=\mathbf{1}_{(-\infty, 0)}(\cdot) & \text { on } \mathbb{R} \times\{0\}\end{cases}
$$

For a given $p$, we define

$$
p_{*}(t)=\liminf _{0 \leq s \rightarrow t} p(s), \quad p^{*}(t)=\limsup _{0 \leq s \rightarrow t} p(s) \quad \forall t \geq 0 .
$$

Since cumulative probability distribution functions (hence $q$ ) are increasing and right continuous, we see that for any $b, p(t)=\mathbb{P}\{\tau>t\} \geq 0$ is decreasing and right continuous, and in particular, $p=p_{*}$. Furthermore, Blumenthal's zero-one law (see, for example [9]) implies that we must have either $p(0)=0$ (in which case the problem is trivial) or $p(0)=1$. Therefore, in the remainder of the paper, we shall only consider lower semicontinuous $p$ for which $p(0)=1$.

For a function $w$ defined on $\mathbb{R} \times[0, \infty)$, we define $w^{*}$ and $w_{*}$ by

$$
\begin{array}{lll}
w^{*}(x, t):=\limsup _{y \rightarrow x, 0 \leq s \rightarrow t} w(y, s), & \forall(x, t) \in \mathbb{R} \times[0, \infty), \\
w_{*}(x, t):=\liminf _{y \rightarrow x, 0 \leq s \rightarrow t} w(y, s), & \forall(x, t) \in \mathbb{R} \times[0, \infty) .
\end{array}
$$

A function $w$ is called upper-semi-continuous (USC) if $w=w^{*}$, and lower-semicontinuous (LSC) if $w=w_{*}$.

In the sequel, the parabolic open ball $B_{\delta}(x, t)$ is defined as:

$$
B_{\delta}(x, t):=(x-\delta, x+\delta) \times\left(t-\delta^{2}, t\right) \quad \forall \delta>0,(x, t) \in \mathbb{R} \times[0, \infty) .
$$

For any cylindrical set of the form $D=(s, t) \times \Omega$ where $0 \leq s<t$ and $\Omega \subseteq \mathbb{R}$, the parabolic boundary is defined to be:

$$
\partial_{p} D=\partial \Omega \times(s, t) \cup \bar{\Omega} \times\{s\}
$$

\section{Definition 1 (Viscosity Sub, Super, and Solutions).}

1. A function $w$ defined on $\mathbb{R} \times(0, \infty)$ is called a (viscosity) subsolution if

$$
w=\min \left\{p, w^{*}\right\} \quad \text { in } \mathbb{R} \times(0, \infty),
$$

and $\mathcal{L} \varphi(x, t) \leq 0$ whenever $\varphi$ is smooth and $w^{*}-\varphi$ attains at $(x, t)$ a local maximum on $\bar{B}_{\delta}(x, t)$, where $x \in \mathbb{R}$ and $t>\delta^{2}>0$. 
2. A function $w$ defined on $\mathbb{R} \times(0, \infty)$ is called a (viscosity) supersolution if

$$
0 \leq w=w_{*} \quad \text { in } \mathbb{R} \times(0, \infty)
$$

and $\max \{w(x, t)-p(t), \quad \mathcal{L} \varphi(x, t)\} \geq 0$ whenever $\varphi$ is smooth and $w-\varphi$ attains at $(x, t)$ a local minimum on $\bar{B}_{\delta}(x, t)$, where $x \in \mathbb{R}$ and $t>\delta^{2}>0$.

3. A function $w$ defined on $\mathbb{R} \times[0, \infty)$ is called a (viscosity) solution if $w$ is both a subsolution and a supersolution in $\mathbb{R} \times(0, \infty)$, and for all $x \in \mathbb{R}$,

$$
w(x, 0)=\liminf _{y \rightarrow x, t \searrow 0} w(y, t)=\mathbf{1}_{(-\infty, 0)}, \quad \limsup _{y \rightarrow x, t \searrow 0} w(y, t)=\mathbf{1}_{(-\infty, 0]} .
$$

Remark 2.1. Here we use the default that a viscosity solution is LSC $\left(w=w_{*}\right)$. Also, the (probabilistically obvious) condition $w \geq 0$ imposed for super-solutions is to ensure the boundedness of the super-solution, as is usually required. This condition could be relaxed to the assumption that $w \geq-e^{A\left(1+|x|^{2}\right)}$ for some $A>0$.

To prove the uniqueness of the solution to (2.2), we first establish a few properties of viscosity solutions.

Lemma 2.1. Let $w$ be a viscosity solution and define

$$
Q:=\{(x, t) \in \mathbb{R} \times[0, \infty) \mid w(x, t)<p(t)\}, \quad \Pi:=Q^{c}=\mathbb{R} \times[0, \infty) \backslash Q .
$$

Then

1. $Q$ is open and $w$ is a smooth solution to $\mathcal{L} w=0$ in $Q$;

2. $\Pi=\{(x, t) \in \mathbb{R} \times[0, \infty) \mid w(x, t)=p(t)\}=\Pi_{0} \cup \Pi_{1} \cup \Pi_{2}$ where

$$
\begin{aligned}
& \Pi_{0}:=\left\{(x, t) \in \mathbb{R} \times[0, \infty) \mid w^{*}(x, t)=w_{*}(x, t)=p(t)\right\}, \\
& \Pi_{1}:=\left\{(x, t) \in \mathbb{R} \times[0, \infty) \mid p^{*}(t)>w^{*}(x, t)>w_{*}(x, t)=p(t)\right\}, \\
& \Pi_{2}:=\left\{(x, t) \in \mathbb{R} \times[0, \infty) \mid p^{*}(t)=w^{*}(x, t)>w_{*}(x, t)=p(t)\right\} .
\end{aligned}
$$

In particular, if $p$ is continuous, then $w$ is continuous in $\mathbb{R} \times[0, \infty) \backslash\{(0,0)\}$.

Proof. 1. First we show that $Q$ is open and $w$ is continuous in $Q$. For any $(x, t) \in Q$, $w(x, t)<p(t)$. As a supersolution, $w(x, t)=w_{*}(x, t)$, and as a subsolution, $w(x, t)=$ 
$\min \left\{p(t), w^{*}(x, t)\right\}=w^{*}(x, t)$. Then $w_{*}=w=w^{*}$ at $(x, t)$. So that $w$ is continuous at $(x, t)$ and $w<p$ in a neighborhood of $(x, t)$. Consequently, $Q$ is open and $w$ is continuous in $Q$.

Next we prove $\mathcal{L} w=0$ in $Q$. Let $\left(x_{0}, t_{0}\right) \in Q$ with $t_{0}>0$. Then $w\left(x_{0}, t_{0}\right)<p\left(t_{0}\right)$ and $w$ is continuous at $\left(x_{0}, t_{0}\right)$. There exist positive constants $\eta$ and $\delta$ such that $w<p-\eta$ in $\bar{D} \subset Q$ where $D=\left(x_{0}-\delta, x_{0}+\delta\right) \times\left(t_{0}-\delta^{2}, t_{0}+\delta^{2}\right)$. Denote by $\tilde{w}$ the solution to $\mathcal{L} \tilde{w}=0$ in $D$ with continuous initial data $\tilde{w}\left(\cdot, t_{0}-\delta^{2}\right)=w\left(\cdot, t_{0}-\delta^{2}\right)$ and boundary data $\tilde{w}=w$ on the parabolic boundary $\partial_{p} D$. Let $\varepsilon>0$ and $\varphi^{\varepsilon}=\tilde{w}-\frac{\varepsilon}{t_{0}+\delta^{2}-t}, \psi^{\varepsilon}=\tilde{w}+\frac{\varepsilon}{t_{0}+\delta^{2}-t}$. We have that $w-\varphi^{\varepsilon}>0$ on $\partial_{p} D$, and $w-\varphi^{\varepsilon} \rightarrow \infty$ as $t \nearrow t_{0}+\delta^{2}$. Suppose there is a point $(x, t) \in D$ such that $w(x, t)-\varphi^{\varepsilon}(x, t) \leq 0$, then $w-\varphi^{\varepsilon}$ will have a local minimum in $D$, say at $\left(x^{*}, t^{*}\right)$. Since $w$ is a supersolution, and $w\left(x^{*}, t^{*}\right)<p\left(t^{*}\right)$ we must have $-\frac{\varepsilon}{t_{0}+\delta^{2}-t^{*}}=\mathcal{L} \varphi^{\varepsilon} \geq 0$, which is a contradiction. Thus $w>\varphi^{\varepsilon}$. A similar argument gives that $w<\psi^{\varepsilon}$. Sending $\varepsilon \rightarrow 0$ we obtain $w=\tilde{w}$ in $D$, which implies that $w$ is a smooth solution to $\mathcal{L} w=0$ in $Q$.

2. Since $w \leq p, \Pi:=Q^{c}$. As a subsolution $w=\min \left\{p, w^{*}\right\} \leq p$, and as a supersolution, $w=w_{*}$. It follows that $w_{*}=w=p \leq w^{*}$ and $w^{*} \leq p^{*}$ in $\Pi$. Hence $w_{*}=p \leq w^{*} \leq p^{*}$. There are only three possibilities for $w^{*}$ : (i) $w^{*}=p$, (ii) $w^{*} \in\left(p, p^{*}\right)$ and (iii) $w^{*}=p^{*}>p$. Thus $\Pi=\Pi_{0} \cup \Pi_{1} \cup \Pi_{2}$.

The following Lemma characterizes the discontinuities of a solution.

Lemma 2.2. Suppose $w$ is a viscosity solution. Then for each $t>0$, the following hold:

1. $w(\cdot, t)=w_{*}(\cdot, t)$ is continuous in $\mathbb{R}$;

2. for each $x \in \mathbb{R}$,

$$
\begin{aligned}
& w_{*}(x, t)=\min \left\{p(t), w^{*}(x, t)\right\}=\lim _{y \rightarrow x, s \backslash t} w(y, s), \\
& w^{*}(x, t)=\lim _{y \rightarrow x, s \nearrow t} w(y, s) \leq p^{*}(t)
\end{aligned}
$$

3. if $w^{*}(x, t)<p^{*}(t)$, then for some $\delta>0, w=w^{*}$ in $B_{\delta}(x, t)$ and $w^{*}$ is a smooth solution to $\mathcal{L} w^{*}=0$ in $\bar{B}_{\delta}(x, t)$. 
Proof. 1. If $w(x, t)<p(t)$, by Lemma (2.1) $w$ is continuous near $(x, t)$; otherwise $w(x, t)=$ $p(t)$. Then using the subsolution property, $\liminf _{y \rightarrow x} w(y, t) \geq w_{*}(x, t)=w(x, t)=p(t) \geq$ $\lim \sup _{y \rightarrow x} w(y, t)$. Thus $\liminf _{y \rightarrow x} w(y, t)=w(x, t)=\limsup _{y \rightarrow x} w(y, t)$, and the first assertion follows.

2. Next we prove (2.4). The first equality is immediate since $w$ is both a subsolution and a supersolution. The second follows by considering separately the cases $w(x, t)<p(t)$ and $w(x, t)=p(t)$ as in the previous step. If $w(x, t)<p(t)$, then $w$ is continuous near $(x, t)$. So that $w_{*}(x, t)=\min \left\{p(t), w^{*}(x, t)\right\}=\lim _{y \rightarrow x, s \backslash t} w(y, s)$. If $w(x, t)=p(t)$, then $w_{*}(x, t)=w(x, t)=p(t) \geq \lim _{y \rightarrow x, s \backslash t} w(y, s) \geq w_{*}(x, t)$. Thus (2.4) holds.

3. Now we prove (2.5) and the third assertion when $w^{*}(x, t)<p^{*}(t)$. By the upper semicontinuity of $w^{*}$, there exist some positive constants $\delta$ and $\eta$ such that $w(\cdot, \cdot)<$ $p^{*}(t)-\eta$ in $\bar{B}_{\delta}(x, t)$. For all $s<t, p(s) \geq p^{*}(t)$. Following the same proof as that for the previous Lemma, we conclude that $w^{*}=w$ in $B_{\delta}(x, t)$ and $w^{*}$ is a smooth solution to $\mathcal{L} w^{*}=0$ in $\bar{B}_{\delta}(x, t)$. The third assertion and $(2.5)$ for the case $w^{*}(x, t)<p^{*}(t)$ thus follow.

4. Finally we verify $(2.5)$ for the case $w^{*}(x, t)=p^{*}(t)$. For each small $\delta>0$, we compare $w$ in $B_{\delta}(x, t)$ with solutions $\bar{w}$ and $\underline{w}$ to

$$
\left\{\begin{array} { l l } 
{ \mathcal { L } \overline { w } = 0 } & { \text { in } B _ { \delta } , } \\
{ \overline { w } = w ^ { * } } & { \text { on } \partial _ { p } B _ { \delta } , }
\end{array} \text { and } \quad \left\{\begin{array}{ll}
\mathcal{L} \underline{w}=0 & \text { in } B_{\delta} \\
\underline{w}=\min \left\{w^{*}, p^{*}(t)\right\} & \text { on } \partial_{p} B_{\delta}
\end{array}\right.\right.
$$

respectively. Note that $w \leq w^{*}=\bar{w}$ and $\underline{w}=\min \left\{w^{*}, p^{*}(t)\right\} \leq \min \left\{w^{*}, p\right\}=w$ on $\partial_{p} B_{\delta}$ since $\underline{w} \leq p^{*}(t) \leq p(s)$ for all $s<t$. By the maximum principle, $\underline{w} \leq w \leq \bar{w}$ in $B_{\delta}$. Observing that $\max _{\bar{B}_{\delta}}\{\bar{w}-\underline{w}\}=\max _{\partial_{p} B_{\delta}}\{\bar{w}-\underline{w}\} \leq p^{*}\left(t-\delta^{2}\right)-p^{*}(t)$, we find

$$
\limsup _{y \rightarrow x, s \nearrow t} w(y, s)-\liminf _{y \rightarrow x, s \nearrow t} w(y, s) \leq \sup _{B_{\delta}(x, t)}\{\bar{w}-\underline{w}\} \leq p^{*}\left(t-\delta^{2}\right)-p^{*}(t) .
$$

(2.5) then follows by sending $\delta \rightarrow 0$.

Theorem 2 (Uniqueness). There is at most one viscosity solution.

Proof. Suppose $w_{1}$ and $w_{2}$ are two solutions. We claim that for any $\eta>0$,

$$
w_{1}(x, t) \leq w_{2}(x-\eta, t) \quad \forall(x, t) \in \mathbb{R} \times[0, \infty) .
$$


To the contrary, suppose this is not true, i.e., there exists at least one pair of $(\bar{x}, \bar{t}) \in$ $\mathbb{R} \times[0, \infty)$ such that $w_{1}(\bar{x}, \bar{t})>w_{2}(\bar{x}-\eta, \bar{t})$. Then for all sufficiently small positive $\varepsilon$,

$$
M_{\varepsilon}:=\sup _{x \in \mathbb{R}, t \geq 0}\left\{w_{1}(x, t)-w_{2}(x-\eta, t)-\varepsilon^{4} x^{2}-\varepsilon e^{t}\right\}>0 .
$$

Hence fix such a positive $\varepsilon$ such that $\varepsilon \leq 1 /\left(1+\left\|\sigma^{2}\right\|_{\infty}+2\left\|\sigma \sigma_{x}-\mu\right\|_{\infty}\right)$. Let $\left\{\left(x_{n}, t_{n}\right)\right\}_{n=1}^{\infty}$ be a sequence in $\mathbb{R} \times[0, \infty)$ such that the supremum $M_{\varepsilon}$ is attained along the sequence. This sequence is bounded since $0 \leq w_{1}, w_{2} \leq 1$. By taking a subsequence if necessary, there exist the limits $(\hat{x}, \hat{t}):=\lim _{n \rightarrow \infty}\left(x_{n}, t_{n}\right), \alpha:=\lim _{n \rightarrow \infty} w_{1}\left(x_{n}, t_{n}\right)$ and $\beta:=\lim _{n \rightarrow \infty} w_{2}\left(x_{n}-\right.$ $\left.\eta, t_{n}\right)$. Note that

$$
\begin{gathered}
0 \leq w_{1 *}(\hat{x}, \hat{t}) \leq \alpha \leq w_{1}^{*}(\hat{x}, \hat{t}) \leq 1 \\
0 \leq w_{2 *}(\hat{x}-\eta, \hat{t}) \leq \beta \leq w_{2}^{*}(\hat{x}-\eta, \hat{t}) \leq 1 \\
\beta<\alpha .
\end{gathered}
$$

Consequently,

$$
M_{\varepsilon}=\alpha-\beta-\varepsilon^{4} \hat{x}^{2}-\varepsilon e^{\hat{t}}>0 .
$$

Additionally, $|\hat{x}|<\varepsilon^{-2}$. Otherwise $M_{\varepsilon} \leq \alpha-\beta-1-\varepsilon \leq-\varepsilon<0$, which is a contradiction to $(2.7)$.

Now we show that this is impossible, by excluding the following three possibilities:
(i) $\hat{t}=0$;
(ii) $\hat{t}>0, \beta<p(\hat{t})$;
(iii) $\hat{t}>0, \beta \geq p(\hat{t})$.

Case (i): Suppose $\hat{t}=0$. If $\hat{x} \geq \eta$, then $0 \leq \beta<\alpha \leq w_{1}^{*}(\hat{x}, 0)=0$; otherwise $1 \geq \alpha>\beta \geq w_{2 *}(\hat{x}-\eta, 0)=1$. Both are impossible.

Case (ii) Suppose $\hat{t}>0$ and $\beta<p(\hat{t})$. Then $w_{2 *}(\hat{x}-\eta, \hat{t}) \leq \beta<p(\hat{t})$. By Lemma (2.1) $w_{2}$ is a smooth solution to $\mathcal{L} w_{2}(\cdot-\eta, \cdot)=0$ in $\bar{D}$ where $D=(\hat{x}-\delta, \hat{x}+\delta) \times\left(\hat{t}-\delta^{2}, \hat{t}+\delta^{2}\right)$ for some $\delta>0$. Let

$$
\varphi(x, t)=w_{2}(x-\eta, t)+\varepsilon^{4} x^{2}+\varepsilon e^{t}+(x-\hat{x})^{4} / \delta^{4}+(t-\hat{t})^{2} / \delta^{4} .
$$

Then $\varphi$ is smooth in $\bar{D}$ and

$$
\max _{D}\left\{w_{1}^{*}-\varphi\right\}=\sup _{\bar{D}}\left\{w_{1}-\varphi\right\} \leq M_{\varepsilon} \leq w_{1}^{*}(\hat{x}, \hat{t})-\varphi(\hat{x}, \hat{t})
$$


That is, $(\hat{x}, \hat{t})$ is a local maximum of $w_{1}^{*}-\varphi$ in $D$. As $w_{1}$ is a subsolution, $\mathcal{L} \varphi(\hat{x}, \hat{t}) \leq 0$. However $\mathcal{L} \varphi(\hat{x}, \hat{t})=\varepsilon e^{\hat{t}}-\varepsilon^{4} \sigma^{2}-2\left(\sigma \sigma_{x}-2 \mu\right) \varepsilon^{4} \hat{x} \geq \varepsilon-\varepsilon^{2}\left\|\sigma^{2}\right\|_{\infty}-2 \varepsilon^{2}\left\|\sigma \sigma_{x}-\mu\right\|_{\infty}>0$ by the smallness of $\varepsilon$. This is a contradiction. Thus case (ii) is impossible.

Case (iii): Suppose $\hat{t}>0$ and $\beta \geq p(\hat{t})$. Since $p^{*}(s) \leq p(t)$ for any $s>\hat{t}, w_{1}(x, s) \leq$ $p(s) \leq p^{*}(t)$ for each $x$. Then $\sup _{x} w_{1}(x, s) \leq p(\hat{t}) \leq \beta<\alpha=\lim _{n \rightarrow \infty} w_{1}\left(x_{n}, t_{n}\right)$. We claim $t_{n}<\hat{t}$ for all sufficiently large $n$, i.e., there exists $N \in \mathbb{N}^{+}$such that $t_{n}<\hat{t}$ for each $n \geq N$. To the contrary, suppose for each $N \in \mathbb{N}^{+}$, there exists $n>N$ such that $t_{n}>\hat{t}$. Then $\alpha>\beta \geq \sup _{x} w_{1}\left(x, t_{n}\right)>w_{1}\left(x_{n}, t_{n}\right)$. Hence there exists $\varepsilon>0$, independent on $n$, such that $\alpha>w_{1}\left(x_{n}, t_{n}\right)+\varepsilon$. This is a contradiction to $\alpha=\lim _{n \rightarrow \infty} w_{1}\left(x_{n}, t_{n}\right)$. Consequently, from (2.5), we conclude that

$$
\alpha=w_{1}^{*}(\hat{x}, \hat{t}) \leq p^{*}(\hat{t}), \quad \beta=w_{2}^{*}(\hat{x}-\eta, \hat{t}) \leq \alpha<p^{*}(\hat{t})
$$

By Lemma $2.2(3)$, for some $\delta>0, w_{2}^{*}=w_{2}$ in $B_{\delta}(\hat{x}+\eta, \hat{t})$ and $w_{2}^{*}$ is a smooth solution to $\mathcal{L} w_{2}^{*}=0$ in $\bar{B}_{\delta}(\hat{x}+\eta, \hat{t})$. Set

$$
\phi(x, t):=w_{2}^{*}(x-\eta, t)+\varepsilon^{4} x^{2}+\varepsilon e^{t}+(x-\hat{x})^{4} / \delta^{4}+(t-\hat{t})^{2} / \delta^{4} .
$$

Then, by $(2.5)$ and $w_{2}^{*}=w_{2}$ in $B_{\delta}(\hat{x}+\eta, \hat{t})$,

$$
\max _{\bar{B}_{\delta}(\hat{x}, \hat{t})}\left\{w_{1}^{*}-\phi\right\}=\sup _{B_{\delta}(\hat{x}, \hat{t})}\left\{w_{1}-\phi\right\} \leq M_{\varepsilon}=w_{1}^{*}(\hat{x}, \hat{t})-\phi(\hat{x}, \hat{t})
$$

That is $w_{1}^{*}-\phi$ obtains its local maximum at $(\hat{x}, \hat{t})$. As $w_{1}$ is a subsolution, $\mathcal{L} \phi(\hat{x}, \hat{t}) \leq 0$. However $\mathcal{L} \phi(\hat{x}, \hat{t}) \varepsilon e^{\hat{t}}-\varepsilon^{4} \sigma^{2}-2\left(\sigma \sigma_{x}-2 \mu\right) \varepsilon^{4} \hat{x} \geq \varepsilon-\varepsilon^{2}\left\|\sigma^{2}\right\|_{\infty}-2 \varepsilon^{2}\left\|\sigma \sigma_{x}-\mu\right\|_{\infty}>0$ by the smallness of $\varepsilon$. This is a contradiction. Thus case (iii) is impossible.

The exclusion of cases (i),(ii) and (iii) implies that (2.6) holds for each $\eta>0$. Sending $\eta \rightarrow 0$ and using Lemma $2.2(1)$ we conclude that $w_{1} \leq w_{2}$ on $\mathbb{R} \times[0, \infty)$. Exchanging the roles of $w_{1}$ and $w_{2}$, we also have $w_{2} \leq w_{1}$, so that $w_{1} \equiv w_{2}$.

As a product, (2.6) and the uniqueness give the following.

Corollary 2.3. The unique solution $w$, if it exists, is non-increasing in $x$, i.e., $w(x, t) \leq$ $w(x-\eta, t)$ for all $\eta>0$ and $(x, t) \in \mathbb{R} \times[0, \infty)$. 


\section{Existence of a Viscosity Solution}

To establish a solution, we first define and study a regularization of the problem obtained by penalizing the obstacle. This $\varepsilon$-regularization is carefully designed so that the solution is monotonic in $\varepsilon$, and therefore the existence of a limit as $\varepsilon \rightarrow 0$ is automatically guaranteed. Then we prove some regularity properties of the solution to the penalized problem for the purpose of establishing compactness. Finally, we show that the limit is a viscosity solution.

\subsection{The Regularization}

Following the classical penalization technique (see for example Friedman [6]) for variational inequalities, we consider a semi-linear parabolic equation:

$$
\begin{cases}\mathcal{L} w^{\varepsilon}=-\beta\left(\varepsilon^{-1}\left(w^{\varepsilon}-p^{\varepsilon}\right)\right) & \text { in } \mathbb{R} \times(0, \infty), \\ w^{\varepsilon}(\cdot, 0)=W^{\varepsilon}(\cdot) & \text { on } \mathbb{R} \times\{0\} .\end{cases}
$$

where $p^{\varepsilon}$ and $W^{\varepsilon}$ are the smooth approximations of $p$ and $w(\cdot, 0)=\mathbf{1}_{(-\infty, 0)}$ respectively, and $\beta(\cdot)$ is a smooth function being identically zero in $(-\infty, 0]$ and strictly increasing and convex in $[0, \infty)$. For definiteness, we take

$$
\beta(s):=\max \left\{0, s^{3}\right\} \quad \forall s \in \mathbb{R} .
$$

The particular $p^{\varepsilon}$ and $W^{\varepsilon}$ are chosen so that the solution $w^{\varepsilon}$ is strictly increasing in $\varepsilon$. Define

$$
p^{\varepsilon}(t):=\frac{3}{4} \int_{-1}^{1}\left(1-z^{2}\right) p(t+\varepsilon+\varepsilon z) d z-3 \varepsilon^{2 / 3} \quad \forall \varepsilon>0, t \geq 0
$$

Then $p^{\varepsilon} \in C^{1}([0, \infty))$, and

$$
-\frac{1}{\varepsilon} \leq \frac{d}{d t} p^{\varepsilon}(t) \leq 0, \quad \frac{d}{d \varepsilon} p^{\varepsilon}(t) \leq-\frac{2}{\varepsilon^{1 / 3}}, \quad \lim _{\varepsilon \backslash 0} p^{\varepsilon}(t)=p(t) \quad \forall \varepsilon>0, t \geq 0 .
$$

The first two inequalities follow directly from the definition of $p^{\varepsilon}$ and monotonicity of $p$. Consequently for any fixed $t>0$, the limit as $\varepsilon \searrow 0$ of $p^{\varepsilon}(t)$ exists, and is $p(t)$.

When $t=0$, (3.2) yields: $\lim _{\varepsilon \backslash 0} p^{\varepsilon}(0)=p(0)=1$ and $p^{\varepsilon}(0)$ is a monotone function of $\varepsilon$. We denote by $\varepsilon^{*}>0$ the unique constant such that $p^{\varepsilon^{*}}(0)=0$, and in the sequel assume $\varepsilon \in\left(0, \varepsilon^{*}\right)$. 
Fix a smooth function $W(\cdot)$ defined on $\mathbb{R}$ that satisfies:

$$
W(x)=0 \forall x \geq 0, \quad W(x)=1 \forall x \leq-1, \quad \dot{W} \leq 0 \forall x \in(-1,0) .
$$

Set

$$
W^{\varepsilon}(x):=p^{\varepsilon}(0) W(x / \varepsilon) \quad \forall x \in \mathbb{R}
$$

Then $W^{\varepsilon}$ is a smooth function satisfying:

$$
\frac{d}{d \varepsilon} W^{\varepsilon}(x) \leq 0, \quad \frac{d}{d x} W^{\varepsilon}(x) \leq 0, \quad W^{\varepsilon}(x)=0 \forall x \geq 0, \quad W^{\varepsilon}(x)=p^{\varepsilon}(0) \forall x \leq-\varepsilon .
$$

Before proving the existence of a solution to problem (3.1), we introduce the following functions.

1. Denote by $w_{0}^{\varepsilon}(x, t)$ the solution to:

$$
\begin{cases}\mathcal{L} w_{0}^{\varepsilon}=0 & \text { in } \mathbb{R} \times(0, \infty), \\ w_{0}^{\varepsilon}(\cdot, 0)=W^{\varepsilon}(\cdot) & \text { on } \mathbb{R} \times\{0\} .\end{cases}
$$

Since the problem for $w_{0}^{\varepsilon}$ is linear, the solution $w_{0}^{\varepsilon}$ can be expressed as

$$
w_{0}^{\varepsilon}(x, t)=\int_{\mathbb{R}} K(x, t ; y, 0) w_{0}^{\varepsilon}(y, 0) d y=p^{\varepsilon}(0) \int_{-\infty}^{0} K(x, t ; y, 0) W(y / \varepsilon) d y
$$

where $K(x, t ; y, s)$ is the fundamental solution associated with the linear operator $\mathcal{L}$. In particular, when $\mathcal{L}=\partial_{t}-\frac{1}{2} \partial_{x x}$, i.e., $\mu \equiv 0$ and $\sigma \equiv 1$,

$$
K(x, t ; y, s)=\Gamma(x-y, t-s), \quad \Gamma(x, t)=\frac{1}{\sqrt{2 \pi t}} e^{-x^{2} / 2 t} .
$$

2. Denote by $\rho^{\varepsilon}$ the solution to:

$$
\begin{cases}\frac{d}{d t} \rho^{\varepsilon}(t)=-\beta\left(\frac{\rho^{\varepsilon}(t)-p^{\varepsilon}(t)}{\varepsilon}\right) & \text { in }(0, \infty), \\ \rho^{\varepsilon}(0)=p^{\varepsilon}(0) & \end{cases}
$$

Comparing the solution $\rho^{\varepsilon}$ with the functions of the form $p^{\varepsilon}+C$ where $C$ is constant, one finds that,

$$
p^{\varepsilon}(t) \leq \rho^{\varepsilon}(t) \leq p^{\varepsilon}(t)+\varepsilon\left\|\dot{p}^{\varepsilon}\right\|_{\infty}^{1 / 3}, \quad \dot{\rho}^{\varepsilon}(t) \leq 0, \quad \forall t \geq 0 .
$$

Now we are ready to prove the existence of a solution to problem (3.1). 
Theorem 3. For each $\varepsilon>0$, problem (3.1) admits a unique smooth $\left(C^{2,1}\right)$ solution in $\mathbb{R} \times[0, \infty)$. The solution is continuously differentiable in $\varepsilon$ and satisfies, for all $\varepsilon>0$ and $(x, t) \in \mathbb{R} \times(0, \infty)$,

$$
\begin{gathered}
w_{0}^{\varepsilon}(x, t)+\rho^{\varepsilon}(t)-\rho^{\varepsilon}(0) \leq w^{\varepsilon}(x, t) \leq \min \left\{\rho^{\varepsilon}(t), w_{0}^{\varepsilon}(x, t)\right\}, \\
w_{x}^{\varepsilon}(x, t)<0, \quad \frac{d}{d \varepsilon} w^{\varepsilon}(x, t)<0 .
\end{gathered}
$$

Consequently, the following limit exists

$$
w(x, t):=\lim _{\varepsilon \backslash 0} w^{\varepsilon}(x, t) \quad \forall(x, t) \in \mathbb{R} \times[0, \infty) .
$$

Proof. 1. First we prove that (3.1) admits a unique smooth solution in $\mathbb{R} \times[0, \infty)$ satisfying (3.4). Since $\mathcal{L} w_{0}^{\varepsilon}+\beta\left(\frac{w_{0}^{\varepsilon}-p^{\varepsilon}}{\varepsilon}\right)=\beta\left(\frac{w_{0}^{\varepsilon}-p^{\varepsilon}}{\varepsilon}\right) \geq 0$, $w_{0}^{\varepsilon}$ is a supersolution. Also, as $\max _{x \in \mathbb{R}} W^{\varepsilon}(x)=p^{\varepsilon}(0)=\rho^{\varepsilon}(0), \rho^{\varepsilon}$ is another supersolution. Hence, $\min \left\{\rho^{\varepsilon}, w_{0}^{\varepsilon}\right\}$ is a supersolution.

Set $\underline{w}^{\varepsilon}:=w_{0}^{\varepsilon}(x, t)+\rho^{\varepsilon}(t)-\rho^{\varepsilon}(0)$. We can compute

$$
\begin{aligned}
& \mathcal{L} \underline{w}^{\varepsilon}+\beta\left(\frac{\underline{w}^{\varepsilon}-p^{\varepsilon}}{\varepsilon}\right) \\
= & \mathcal{L} w_{0}^{\varepsilon}(x, t)+\mathcal{L} \rho^{\varepsilon}(t)+\beta\left(\frac{\underline{w}^{\varepsilon}-p^{\varepsilon}}{\varepsilon}\right) \\
= & -\left[\beta\left(\frac{\rho^{\varepsilon}-p^{\varepsilon}}{\varepsilon}\right)-\beta\left(\frac{\rho^{\varepsilon}-p^{\varepsilon}+w_{0}^{\varepsilon}-\rho^{\varepsilon}(0)}{\varepsilon}\right)\right] \leq 0
\end{aligned}
$$

since $\beta(\cdot)$ is non-decreasing and

$$
w_{0}^{\varepsilon} \leq \max \left\{w_{0}^{\varepsilon}(\cdot, 0)\right\}=\max _{x \in \mathbb{R}} W^{\varepsilon}(x)=\max _{x \in \mathbb{R}} p^{\varepsilon}(0) W(x / \varepsilon)=p^{\varepsilon}(0)=\rho^{\varepsilon}(0) .
$$

Hence $\underline{w}^{\varepsilon}$ is a subsolution, and $\underline{w}^{\varepsilon} \leq \min \left\{\rho^{\varepsilon}, w_{0}^{\varepsilon}\right\}$ followed by (3.5). We see that (3.1) admits a unique smooth solution in $\mathbb{R} \times[0, \infty)$ and the solution satisfies (3.4).

2. Differentiating the system (3.1) with respect to $\varepsilon$ we obtain

$$
\begin{aligned}
\frac{d}{d \varepsilon} w^{\varepsilon}(x, 0) & =\frac{d}{d \varepsilon} W^{\varepsilon}(x) \leq 0 \quad \forall x \in \mathbb{R}, \\
\mathcal{L} \frac{d}{d \varepsilon} w^{\varepsilon}+\frac{1}{\varepsilon} \dot{\beta}\left(\frac{w^{\varepsilon}-p^{\varepsilon}}{\varepsilon}\right) \frac{d}{d \varepsilon} w^{\varepsilon} & =\frac{1}{\varepsilon^{2}} \dot{\beta}\left(\frac{w^{\varepsilon}-p^{\varepsilon}}{\varepsilon}\right)\left\{w^{\varepsilon}-p^{\varepsilon}+\varepsilon \frac{d}{d \varepsilon} p^{\varepsilon}\right\} \leq 0,
\end{aligned}
$$


since $\dot{\beta} \geq 0, w^{\varepsilon}-p^{\varepsilon} \leq \rho^{\varepsilon}-p^{\varepsilon} \leq \varepsilon\left\|\dot{p}^{\varepsilon}\right\|_{\infty}^{1 / 3} \leq \varepsilon^{2 / 3}$, and $\frac{d}{d \varepsilon} p^{\varepsilon} \leq-2 \varepsilon^{-1 / 3}$. Then, by the maximum principle, $\frac{d}{d \varepsilon} w^{\varepsilon}<0$ in $\mathbb{R} \times(0, \infty)$. The monotonicity and boundedness of $w^{\varepsilon}$ in $\varepsilon$ and imply that $w=\lim _{\varepsilon \searrow 0} w^{\varepsilon}$ exists.

In a similar manner, differentiating the system (3.1) with respect to $x$ and let $u^{\varepsilon}:=-w_{x}^{\varepsilon}$, we obtain

$$
\begin{gathered}
\mathcal{A} u^{\varepsilon}+\frac{1}{\varepsilon} \dot{\beta}\left(\frac{w^{\varepsilon}-p^{\varepsilon}}{\varepsilon}\right) u^{\varepsilon}=0, \\
u^{\varepsilon}(x, 0)=-\frac{d}{d x} w^{\varepsilon}(x, 0)=-\frac{d}{d x} W^{\varepsilon}(x) \geq 0 \quad \forall x \in \mathbb{R} .
\end{gathered}
$$

where $\mathcal{A} u=\mathcal{L} u-\sigma \sigma_{x} u_{x}+\left(\mu_{x}-\sigma \sigma_{x x}+\left(\sigma_{x}\right)^{2}\right) u$. Since $\frac{1}{\varepsilon} \dot{\beta}\left(\frac{w^{\varepsilon}-p^{\varepsilon}}{\varepsilon}\right)>0,-w_{x}^{\varepsilon}(x, t)=$ $u^{\varepsilon}(x, t)>0$ in $\mathbb{R} \times(0, \infty)$.

Also note that since $w_{0}^{\varepsilon}$ is monotonic in $\varepsilon$ and bounded, the limit $w_{0}:=\lim _{\varepsilon}{ }_{0} w_{0}^{\varepsilon}$ exists and is the solution to

$$
\mathcal{L} w_{0}=0 \text { in } \mathbb{R} \times(0, \infty), \quad w_{0}(\cdot, 0)=\mathbf{1}_{(-\infty, 0)}
$$

\subsection{Continuity Estimates and Existence.}

In this section, we prove that the $\operatorname{limit} w=\lim _{\varepsilon \backslash 0}$ is the viscosity solution to our variational inequality. In order to do so, we first need to derive some supplementary estimates on the continuity of $w$.

Lemma 3.1. For each $T>0$, there exists a constant $C=C(T)$ that depends only on $\sigma$ and $\mu$ such that for all $\varepsilon \in\left(0, \varepsilon^{*}\right), 0<s<t \leq T$, and $x, y \in \mathbb{R}$,

$$
\begin{gathered}
-\frac{C p^{\varepsilon}(0)}{\sqrt{t}} \leq w_{0 x}^{\varepsilon}(x, t) \leq w_{x}^{\varepsilon}(x, t) \leq 0, \\
\left|w^{\varepsilon}(x, t)-w^{\varepsilon}(y, s)\right| \leq \frac{C p^{\varepsilon}(0)}{\sqrt{s}}\{|x-y|+2 \sqrt{t-s}\}+\rho^{\varepsilon}(s)-\rho^{\varepsilon}(t) .
\end{gathered}
$$

Consequently, the limit $w=\lim _{\varepsilon \backslash 0} w^{\varepsilon}$ satisfies for all $0<s<t \leq T$ and $x, y \in \mathbb{R}$,

$$
\begin{gathered}
-\frac{C}{\sqrt{t}} \leq w_{0 x}(x, t) \leq w_{x}(x, t) \leq 0, \\
|w(x, t)-w(y, s)| \leq \frac{C}{\sqrt{s}}\{|x-y|+2 \sqrt{t-s}\}+p(s)-p(t), \\
w(x, t)-w(y, s) \leq \frac{C}{\sqrt{s}}\{|x-y|+2 \sqrt{t-s}\} .
\end{gathered}
$$


We remark that when $\sigma \equiv 1$ and $\mu \equiv 0, C=C(T)=(2 \pi)^{-1 / 2}$ for all $T$.

Proof. Differentiating the systems (3.1) and (3.3) with respect to $x$, and using the notation from the previous theorem, we find

$$
\begin{array}{rc}
\mathcal{A} w_{x}^{\varepsilon}=-\varepsilon^{-1} \dot{\beta}\left(\varepsilon^{-1}\left(w^{\varepsilon}-p^{\varepsilon}\right) w_{x}^{\varepsilon} \geq 0=\mathcal{A} w_{0 x}^{\varepsilon}\right. & \text { in } \mathbb{R} \times(0, \infty), \\
w_{x}^{\varepsilon}(\cdot, 0)=W_{x}^{\varepsilon}(\cdot)=w_{0 x}^{\varepsilon}(\cdot, 0) & \text { on } \mathbb{R} \times\{0\} .
\end{array}
$$

since $w_{x}^{\varepsilon} \leq 0$. Therefore by the maximum principle (the zeroth order term in $\mathcal{A}$ is bounded above) $w_{0 x}^{\varepsilon} \leq w_{x}^{\varepsilon} \leq 0$.

Next we estimate the lower bound of $w_{0 x}^{\varepsilon}$. Differentiating the system (3.3) with respect to $x$, we obtain

$$
\mathcal{A} w_{0 x}^{\varepsilon}=0 \text { in } \mathbb{R} \times(0, \infty), \quad w_{0 x}^{\varepsilon}(\cdot, 0)=W_{x}^{\varepsilon}(\cdot) \text { on } \mathbb{R} \times\{0\} .
$$

This is a linear problem, the solution can be expressed as

$$
w_{0 x}^{\varepsilon}(x, t)=\int_{\mathbb{R}} \tilde{K}(x, t ; y, 0) W_{y}^{\varepsilon}(y) d y,
$$

where $\tilde{K}$ is the fundamental solution associated with the linear operator $\mathcal{A}$, and we therefore have $\tilde{K}>0$. Also $W_{y}^{\varepsilon} \leq 0$, then for any $0<t \leq T$,

$$
0 \leq-w_{0 x}^{\varepsilon}(x, t) \leq \sup _{x, y \in \mathbb{R}}\{\tilde{K}(x, t ; y, 0)\} \int_{\mathbb{R}}-W_{y}^{\varepsilon}(y) d y=\frac{C}{\sqrt{t}} \int_{\mathbb{R}}-W_{y}^{\varepsilon}(y) d y=\frac{C p^{\varepsilon}(0)}{\sqrt{t}}
$$

where

$$
C=C(T)=\sup _{x, y \in \mathbb{R}, 0<t<T}\{\sqrt{t} \tilde{K}(x, t ; y, 0)\} .
$$

and the above quantity is finite by the standard Gaussian upper bound on the fundamental solution $\tilde{K}$ (see Friedman [5]). The estimates for $w_{x}^{\varepsilon}$ and $w_{0 x}^{\varepsilon}$ (3.6) thus follow. Sending $\varepsilon \rightarrow 0$, we obtain (3.8).

Now we estimate the continuity in the time variable. By Theorem $3, w_{x}^{\varepsilon}<0$ and $w^{\varepsilon}(x, t) \geq$ $\rho^{\varepsilon}(t)-\rho^{\varepsilon}(0)$ (since $w_{0}^{\varepsilon} \geq 0$ ). We conclude that $\lim _{x \rightarrow \infty} w^{\varepsilon}$ exists. Similarly, the limit $\lim _{x \rightarrow \infty} w_{0}^{\varepsilon}$ exists, and is nonnegative. Now using $w^{\varepsilon}(x, t) \leq \rho^{\varepsilon}(t)$ for any $t \geq 0$, we can compute

$$
\begin{aligned}
\int_{\mathbb{R}}\left|w_{x}^{\varepsilon}(x, t)\right| d x & =\int_{\mathbb{R}}-w_{x}^{\varepsilon}(x, t) \leq \rho^{\varepsilon}(t)-\lim _{x \rightarrow \infty} w^{\varepsilon}(x, t) \\
& \leq \rho^{\varepsilon}(t)-\lim _{x \rightarrow \infty} w_{0}^{\varepsilon}(x, t)-\rho^{\varepsilon}(t)+\rho^{\varepsilon}(0) \\
& =\rho^{\varepsilon}(0)-\lim _{x \rightarrow \infty} w_{0}^{\varepsilon}(x, t) \leq \rho^{\varepsilon}(0) .
\end{aligned}
$$


Also note that since $\beta(\cdot)$ is increasing and $w^{\varepsilon} \leq \rho^{\varepsilon}$,

$$
0 \leq \beta\left(\varepsilon^{-1}\left(w^{\varepsilon}-p^{\varepsilon}\right)\right) \leq \beta\left(\varepsilon^{-1}\left(\rho^{\varepsilon}-p^{\varepsilon}\right)\right)=-\dot{\rho}^{\varepsilon}(t) \quad \forall t \geq 0, x \in \mathbb{R} .
$$

For $0<s<t \leq T$ denote $\left\|w_{x}^{\varepsilon}\right\|_{\infty}^{s, t}=\sup _{\mathbb{R} \times[s, t]}\left|w_{x}^{\varepsilon}\right|$, then for each $\delta>0$,

$$
\begin{aligned}
& \left|\int_{x-\delta}^{x+\delta}\left\{w^{\varepsilon}(y, t)-w^{\varepsilon}(y, s)\right\} d y\right|=\left|\int_{x-\delta}^{x+\delta} \int_{s}^{t} w_{v}^{\varepsilon}(y, v) d v d y\right| \\
& \quad=\left|\int_{s}^{t} \int_{x-\delta}^{x+\delta}\left(\frac{1}{2}\left(\sigma^{2} w_{y}^{\varepsilon}\right)_{y}-\mu w_{y}^{\varepsilon}-\beta\left(\varepsilon^{-1}\left(w^{\varepsilon}-p^{\varepsilon}\right)\right)\right) d y d v\right| \\
& \leq\left|\int_{s}^{t} \frac{1}{2} \sigma^{2} w_{y}^{\varepsilon}\right|_{x-\delta}^{x+\delta} d v|+| \int_{s}^{t} \int_{x-\delta}^{x+\delta} \mu w_{y}^{\varepsilon} d y d v|+| \int_{s}^{t} \int_{x-\delta}^{x+\delta} \beta\left(\varepsilon^{-1}\left(\rho^{\varepsilon}-p^{\varepsilon}\right)\right) d y d v \mid \\
& \leq(t-s)\left(\left\|\sigma^{2}\right\|_{\infty}\left\|w_{x}^{\varepsilon}\right\|_{\infty}^{s, t}+p^{\varepsilon}(0)\|\mu\|_{\infty}\right)+\left|\int_{s}^{t} \int_{x-\delta}^{x+\delta}-\frac{d}{d v} \rho^{\varepsilon}(v) d y d v\right| \\
& \leq(t-s)\left(\left\|\sigma^{2}\right\|_{\infty}\left\|w_{x}^{\varepsilon}\right\|_{\infty}+p^{\varepsilon}(0)\|\mu\|_{\infty}\right)+2 \delta\left(\rho^{\varepsilon}(s)-\rho^{\varepsilon}(t)\right) .
\end{aligned}
$$

Finally, note that for any $s \geq 0$,

$$
\begin{aligned}
& \left|w^{\varepsilon}(x, s)-\frac{1}{2 \delta} \int_{x-\delta}^{x+\delta} w^{\varepsilon}(y, s) d y\right|=\left|\frac{1}{2 \delta} \int_{x-\delta}^{x+\delta}\left(w^{\varepsilon}(x, s)-w^{\varepsilon}(y, s)\right) d y\right| \\
\leq & \frac{1}{2 \delta} \int_{x-\delta}^{x+\delta}|y-x| \cdot\left\|w_{x}^{\varepsilon}(\cdot, s)\right\|_{\infty} d y \leq \delta\left\|w_{x}^{\varepsilon}(\cdot, s)\right\|_{\infty} .
\end{aligned}
$$

Now we are ready to estimate the continuity in the time variable. For any $0<s<t \leq T$,

$$
\begin{aligned}
& \left|w^{\varepsilon}(x, t)-w^{\varepsilon}(x, s)\right| \\
\leq & \left|w^{\varepsilon}(x, t)-\frac{1}{2 \delta} \int_{x-\delta}^{x+\delta} w^{\varepsilon}(y, t) d y\right|+\left|\frac{1}{2 \delta} \int_{x-\delta}^{x+\delta}\left(w^{\varepsilon}(y, t)-w^{\varepsilon}(y, s)\right) d y\right| \\
& +\left|\frac{1}{2 \delta} \int_{x-\delta}^{x+\delta} w^{\varepsilon}(y, s) d y-w^{\varepsilon}(x, s) d y\right| \\
\leq & \left\|w_{x}^{\varepsilon}\right\|_{\infty}^{s, t}\left(2 \delta+\frac{(t-s)\left\|\sigma^{2}\right\|_{\infty}}{2 \delta}\right)+\frac{(t-s) 2\|\mu\|_{\infty} p^{\varepsilon}(0)}{2 \delta}+\rho^{\varepsilon}(s)-\rho^{\varepsilon}(t) .
\end{aligned}
$$

By taking $\delta=\frac{1}{2} \sqrt{\left\|\sigma^{2}\right\|_{\infty}(t-s)}$, we then obtain

$$
\left|w^{\varepsilon}(x, t)-w^{\varepsilon}(x, s)\right| \leq \sqrt{\left\|\sigma^{2}\right\|_{\infty}(t-s)}\left(2\left\|w_{x}^{\varepsilon}\right\|_{\infty}^{s, t}+\frac{2\|\mu\|_{\infty} p^{\varepsilon}(0)}{\left\|\sigma^{2}\right\|_{\infty}}\right)+\rho^{\varepsilon}(s)-\rho^{\varepsilon}(t) .
$$

Since $0 \geq w_{x}^{\varepsilon}(x, s) \geq-\frac{C p^{\varepsilon}(0)}{\sqrt{s}}$, we have

$$
\left|w^{\varepsilon}(x, s)-w^{\varepsilon}(y, s)\right| \leq|x-y| \cdot\left\|w_{x}^{\varepsilon}(\cdot, s)\right\|_{\infty} \leq \frac{C p^{\varepsilon}(0)}{\sqrt{s}}|x-y|
$$


Similarly, $\left\|w_{x}^{\varepsilon}\right\|_{\infty}^{s, t} \leq \frac{C p^{\varepsilon}(0)}{\sqrt{s}}$. Then

$$
\begin{aligned}
& \left|w^{\varepsilon}(x, t)-w^{\varepsilon}(y, s)\right| \leq\left|w^{\varepsilon}(x, t)-w^{\varepsilon}(x, s)\right|+\left|w^{\varepsilon}(x, s)-w^{\varepsilon}(y, s)\right| \\
\leq & \frac{C p^{\varepsilon}(0)}{\sqrt{s}}\{|x-y|+2 \sqrt{t-s}\}+\rho^{\varepsilon}(s)-\rho^{\varepsilon}(t) .
\end{aligned}
$$

This proves (3.7) and (3.9) then follows by sending $\varepsilon \rightarrow 0$. Finally, observe that in estimating the upper bound of $w^{\varepsilon}(x, s)-w^{\varepsilon}(x, t)$, the term involving the integral of $\beta$ can be dropped, so we have (3.10). This completes the proof.

We can now show the following:

Theorem 4. Assume $p(\cdot)$ defined on $[0, \infty)$ is nonnegative, decreasing and lower semicontinuous, with $p(0)=1$. Then there is a unique viscosity solution, and it can be obtained as the limit $w:=\lim _{\varepsilon \backslash 0} w^{\varepsilon}$.

Proof. First we verify that $w$ satisfies the initial condition (2.3). For any $t>0$, from (3.4) $\left|w^{\varepsilon}(\cdot, t)-w_{0}^{\varepsilon}(\cdot, t)\right| \leq \rho^{\varepsilon}(0)-\rho^{\varepsilon}(t)$, so that

$$
\left\|w(\cdot, t)-w_{0}(\cdot, t)\right\|_{\infty} \leq \lim _{\varepsilon \backslash 0}\left\|w^{\varepsilon}(\cdot, t)-w_{0}^{\varepsilon}(\cdot, t)\right\|_{\infty} \leq p(0)-p(t)
$$

where

$$
\begin{aligned}
& w_{0}(x, t)=\lim _{\varepsilon \rightarrow 0} w_{0}^{\varepsilon}(x, t)=\int_{-\infty}^{0} K(x, t ; y, 0) d y, \\
& \left\|w(\cdot, t)-w_{0}(\cdot, t)\right\|_{\infty}=\sup _{x \in \mathbb{R}}\left|w(\cdot, t)-w_{0}(\cdot, t)\right| .
\end{aligned}
$$

Sending $t \searrow 0$, we see that $w$ satisfies (2.3).

To verify that $w$ is a viscosity solution in $\mathbb{R} \times(0, \infty)$ we consider two cases for each $(x, t) \in \mathbb{R} \times(0, \infty):($ i) $p(t)-w(x, t)>0$ and (ii) $p(t)-w(x, t) \leq 0$.

Case (i): Suppose $p(t)-w(x, t)>0$. Let $D_{\delta}=(x-\delta, x+\delta) \times\left(t-\delta^{2}, t+\delta^{2}\right) \forall \delta>0$. Then for each $(y, s) \in D_{\delta}$,

$\rho^{\varepsilon}(s)-\rho^{\varepsilon}(t)|\leq| \rho^{\varepsilon}(s)-p^{\varepsilon}(s)|+| p^{\varepsilon}(s)-p^{\varepsilon}(t)|+| p^{\varepsilon}(t)-\rho^{\varepsilon}(t)|\leq| p^{\varepsilon}(s)-p^{\varepsilon}(t) \mid+2 \varepsilon^{2 / 3}$, since $0 \leq \rho^{\varepsilon}-p^{\varepsilon} \leq \varepsilon^{2 / 3}$. As $p^{\varepsilon}(\cdot)$ is decreasing, when $s>t$,

$$
p^{\varepsilon}(t)-p^{\varepsilon}(s)+\left|p^{\varepsilon}(t)-p^{\varepsilon}(s)\right|=2\left(p^{\varepsilon}(t)-p^{\varepsilon}(s)\right) \leq 2\left(p^{\varepsilon}(t)-p^{\varepsilon}\left(t+\delta^{2}\right)\right)
$$


and when $s \leq t$,

$$
p^{\varepsilon}(t)-p^{\varepsilon}(s)+\left|p^{\varepsilon}(t)-p^{\varepsilon}(s)\right|=0 \leq 2\left(p^{\varepsilon}(t)-p^{\varepsilon}\left(t+\delta^{2}\right)\right) .
$$

Using (3.7), we can compute

$$
\begin{aligned}
w^{\varepsilon}(y, s)-p^{\varepsilon}(s) & \leq w^{\varepsilon}(x, t)+\frac{(2+2 \sqrt{2}) C \delta}{\sqrt{t-\delta^{2}}}+\left|\rho^{\varepsilon}(s)-\rho^{\varepsilon}(t)\right|-p^{\varepsilon}(s) \\
& \leq \frac{(2+2 \sqrt{2}) C \delta}{\sqrt{t-\delta^{2}}}+w^{\varepsilon}(x, t)-p^{\varepsilon}(t)+p^{\varepsilon}(t)-p^{\varepsilon}(s)+\left|p^{\varepsilon}(t)-p^{\varepsilon}(s)\right|+2 \varepsilon^{2 / 3} \\
& \leq \frac{(2+2 \sqrt{2}) C \delta}{\sqrt{t-\delta^{2}}}+w^{\varepsilon}(x, t)-p^{\varepsilon}(t)+2\left(p^{\varepsilon}(t)-p^{\varepsilon}\left(t+\delta^{2}\right)\right)+2 \varepsilon^{2 / 3} .
\end{aligned}
$$

Then if we take $\delta$ small enough,

$$
\begin{aligned}
\limsup _{\varepsilon \rightarrow 0} \max _{\bar{D}_{\delta}}\left\{w^{\varepsilon}-p^{\varepsilon}\right\} & \leq \limsup _{\varepsilon \rightarrow 0} \frac{(2+2 \sqrt{2}) C \delta}{\sqrt{t-\delta^{2}}}+w^{\varepsilon}(x, t)-p^{\varepsilon}(t)+2\left(p^{\varepsilon}(t)-p^{\varepsilon}\left(t+\delta^{2}\right)\right)+2 \varepsilon^{2 / 3} \\
& \leq \frac{(2+2 \sqrt{2}) C \delta}{\sqrt{t-\delta^{2}}}+w(x, t)-p(t)+2\left(p(t)-p\left(t+\delta^{2}\right)\right)<0 .
\end{aligned}
$$

Thus, for all sufficiently small positive $\varepsilon, w^{\varepsilon}-p^{\varepsilon}<0$ in $\bar{D}_{\delta}$. Consequently, $\mathcal{L} w^{\varepsilon}=$ $-\beta\left(\frac{w^{\varepsilon}-p^{\varepsilon}}{\varepsilon}\right)=0$ in $\bar{D}_{\delta}$. The limit $w$ is then a smooth solution to $\mathcal{L} w=0$ in $D_{\delta}$.

Case (ii): Suppose $w(x, t)-p(t) \geq 0$. However, $w-p \leq 0$ in $\mathbb{R} \times[0, \infty)$ since $w^{\varepsilon} \leq$ $\rho^{\varepsilon}$ and $\lim _{\varepsilon \backslash 0} \rho^{\varepsilon}(t)=p(t)$ in $\mathbb{R} \times[0, \infty)$. Hence, we must have $w(x, t)=p(t)=$ $\min \left\{p(t), w^{*}(x, t)\right\}$. From $(3.10)$

$$
w(x, t)-w_{*}(x, t)=\limsup _{y \rightarrow x, s \rightarrow t}(w(x, t)-w(y, s)) \leq \limsup _{y \rightarrow x, s \rightarrow t}\left(\frac{C}{\sqrt{s}}\{|x-y|+2 \sqrt{t-s}\}\right)=0 .
$$

So that $w_{*}(x, t)=w(x, t)=p(t)$. Thus the semi-continuity requirements for a viscosity solution hold.

In this case, we clearly have $\max \{w(x, t)-p(t), \mathcal{L} \varphi(x, t)\} \geq 0$ for any smooth $\varphi$. So that $w$ is a supersolution. It remains to verify the differential inequality for subsolutions. To this end, let $\varphi$ be a smooth function on $\bar{B}_{\delta}$ where $B_{\delta}=B_{\delta}(x, t)$ such that $w^{*}(y, s)-$ $\varphi(y, s)$ attains at $(x, t)$ a local maximum on $\bar{B}_{\delta}$. Set

$$
\psi(y, s)=\varphi(y, s)+(y-x)^{4} / \delta^{4}+(s-t)^{2} / \delta^{4} .
$$

For each small positive $\varepsilon, w^{\varepsilon}-\psi$ attains a global maximum on $\bar{B}_{\delta}$. Denote any such point of maximum by $\left(y_{\varepsilon}, s_{\varepsilon}\right)$. Then $\left(w^{\varepsilon}-\psi\right)_{s} \geq 0,\left(w^{\varepsilon}-\psi\right)_{y y} \leq 0,\left(w^{\varepsilon}-\psi\right)_{y}=0$ at 
$\left(y_{\varepsilon}, s_{\varepsilon}\right)$. Thus, $\mathcal{L} \psi\left(y_{\varepsilon}, s_{\varepsilon}\right) \leq \mathcal{L} w^{\varepsilon}\left(y_{\varepsilon}, s_{\varepsilon}\right)=-\beta\left(\varepsilon^{-1}\left(w^{\varepsilon}-p^{\varepsilon}\right)\right) \leq 0$. If $(\bar{x}, \bar{t})$ is a limit point of $\left\{\left(y_{\varepsilon}, s_{\varepsilon}\right)\right\}$ as $\varepsilon \rightarrow 0$, then $\mathcal{L} \psi(\bar{x}, \bar{t}) \leq 0$. Thus, it suffices to show that $(\bar{x}, \bar{t})=(x, t)$.

Since $w^{\varepsilon} \leq w \leq w^{*}$

$$
\begin{aligned}
\limsup _{\varepsilon \backslash 0} \max _{\bar{B}_{\delta}}\left\{w^{\varepsilon}-\psi\right\} & \leq \limsup _{\varepsilon \searrow 0}\left\{w^{*}\left(y_{\varepsilon}, s_{\varepsilon}\right)-\psi\left(y_{\varepsilon}, s_{\varepsilon}\right)\right\} \\
& \leq w^{*}(\bar{x}, \bar{t})-\psi(\bar{x}, \bar{t}) \leq \max _{\bar{B}_{\delta}}\left(w^{*}-\varphi\right)-|\bar{x}-x|^{4} / \delta^{4}-|\bar{t}-t|^{2} / \delta^{4} .
\end{aligned}
$$

On the other hand, from (3.9) and (3.10), we see that $w^{*}(\hat{x}, t)=\lim _{s / t} w(\hat{x}, s)$, so that

$$
\begin{aligned}
\limsup _{\varepsilon \searrow 0} \max _{\bar{B}_{\delta}}\left\{w^{\varepsilon}-\psi\right\} & \geq \lim _{s \nearrow t} \limsup _{\varepsilon \searrow 0}\left\{w^{\varepsilon}(x, s)-\psi(x, s)\right\} \\
& =\lim _{s \nearrow t}\{w(x, s)-\psi(x, s)\}=w^{*}(x, t)-\psi(x, t)=\max _{\bar{B}_{\delta}}\left(w^{*}-\varphi\right) .
\end{aligned}
$$

Thus, we must have $(\bar{x}, \bar{t})=(x, t)$. This completes the proof.

\subsection{The Differential Equation and the Free Boundary Problem}

Since

$$
0 \leq \beta\left(\varepsilon^{-1}\left(w^{\varepsilon}-p^{\varepsilon}\right)\right) \leq-\dot{\rho}^{\varepsilon},
$$

and $\rho^{\varepsilon}(\cdot)$ is decreasing, by weak compactness of measures, as $\varepsilon \rightarrow 0$,

$$
\begin{array}{rll}
\beta\left(\varepsilon^{-1}\left(w^{\varepsilon}-p^{\varepsilon}\right)\right) & \longrightarrow \gamma & \text { as a measure in } \mathbb{R} \times[0, \infty), \\
\mathcal{L} w & =\gamma & \text { on } \mathbb{R} \times(0, \infty),
\end{array}
$$

where $\gamma$ is a Radon measure satisfying

$$
0 \leq \gamma d x d t \leq-d x d p(t)
$$

In addition, from step 2 of the proof in the preceding subsection, $\gamma$ is supported on the set $w=p$.

Now suppose that $p$ is continuous. Then $\gamma=\dot{p}$ on the contact set $\Pi$ (noticing that $\Pi_{2}$ is empty). Hence, $w$ is the solution to

$$
\mathcal{L} w=\dot{p}(t) \mathbf{1}_{\{w=p\}} \text { in } \mathbb{R} \times(0, \infty), \quad w(\cdot, 0)=\mathbf{1}_{(-\infty, 0)} \text { on } \mathbb{R} \times\{0\}
$$


Using a free boundary approach, this can be written as the solution to the free boundary problem, for $(b, w)$ :

$$
\begin{cases}\mathcal{L} w=\dot{p}(t) \mathbf{1}_{x<b(t)} & \text { in } \mathbb{R} \times(0, \infty), \\ b(t):=\inf \{x \mid w(x, t)<p(t)\} & \text { for all } t \geq 0, \\ w(\cdot, 0)=\mathbf{1}_{(-\infty, 0)} & \text { on } \mathbb{R} \times\{0\} .\end{cases}
$$

We emphasize that this formulation works only when $p$ is continuous, since if $p$ is not continuous at $s$, then

$$
\mathcal{L} w=\min \left\{p(s)-w^{*}(x, \tau), 0\right\} \cdot \delta(t-s) \quad \text { on } \mathbb{R} \times\{s\},
$$

where $\delta$ is the Dirac measure.

Remark 3.1. Suppose $\|\dot{p}\|_{\infty}:=\sup _{t \geq 0}|\dot{p}(t)|$ is finite. Then $\left\|\dot{p}_{\varepsilon}\right\|_{\infty} \leq\|\dot{p}\|_{\infty}$ and $\rho^{\varepsilon}-p^{\varepsilon} \leq$ $\varepsilon\|\dot{p}\|^{1 / 3}$. Consequently, $\dot{\rho}^{\varepsilon}=-\beta\left(\|\dot{p}\|^{1 / 3}\right)=-\|\dot{p}\|_{\infty}$. Hence

$$
0 \leq \gamma^{\varepsilon}(x, t) \leq\|\dot{p}\|_{\infty} \quad \forall(x, t) \in \mathbb{R} \times[0, \infty)
$$

It is then easy to show that $w^{\varepsilon}(x, t)-w_{0}^{\varepsilon} \rightarrow w-w_{0}$ in $W_{r}^{2,1}\left([-R, R] \times\left[0, R^{2}\right]\right)$ for any $r>1$ and any $R>0$.

\section{Estimation of The Free Boundary}

In this section, we provide both upper and lower bounds for the free boundary

$$
b(t):=\inf \{x \in \mathbb{R} \mid w(x, t)<p(t)\} \in[-\infty, \infty] \quad \forall t>0 .
$$

in the case of Brownian motion, i.e. when $\sigma \equiv 1$ and $\mu \equiv 0$.

Recall the notation $q(t)=1-p(t)$. Note that for any $s>0,0=q(0)=q^{*}(0) \leq q(s)$, and since $p$ is lower semicontinuous, $q$ is upper semicontinuous. We define

$$
\dot{q}(s):=\liminf _{t / s} \frac{q(s)-q(t)}{s-t} \in[0, \infty] .
$$

The following lemma is obvious from the probabilistic interpretation of our problem since it states that $\mathbb{P}\left[X_{t} \leq b(t)\right] \leq \mathbb{P}[\tau \leq t]$. Its analytic derivation is equally simple.

Lemma 4.1. For every $t>0, \frac{1}{\sqrt{\pi}} \int_{-\infty}^{b(t) / \sqrt{2 t}} e^{-z^{2}} d z \leq q(t)$. 
Proof. We need only consider the case $b(t)>-\infty$. Since $w(x, t) \leq w_{0}(x, t)$,

$$
\begin{aligned}
1-q(t) & =p(t)=w(b(t), t) \leq w_{0}(b(t), t)=\int_{-\infty}^{0} \Gamma(b(t)-y, t) d y \\
& =1-\frac{1}{\sqrt{2 \pi t}} \int_{0}^{\infty} e^{-\frac{(b(t)-y)^{2}}{2 t}} d y=1-\frac{1}{\sqrt{\pi}} \int_{-\infty}^{b(t) / \sqrt{2 t}} e^{-z^{2}} d z .
\end{aligned}
$$

Lemma 4.2 (Method for Lower Bounds). Assume that $\underline{w}$ defined on $\mathbb{R} \times[0, t]$ satisfies

$$
\begin{cases}\mathcal{L} \underline{w}=0 & \text { in } \mathbb{R} \times(0, t], \\ \underline{w}(\cdot, 0) \leq w(\cdot, 0) & \text { on } \mathbb{R} \times\{0\}, \\ \underline{w} \leq p & \text { on } \mathbb{R} \times(0, t), \\ \underline{w}(s, t) \geq p(t) & \text { at }(s, t) .\end{cases}
$$

Then

$$
\underline{w} \leq w \text { in } \mathbb{R} \times[0, t), \quad s \leq b(t) .
$$

Proof. First consider the case where $p$ is continuous at $t$, so $p(t)=p^{*}(t)$. For each $\varepsilon>0$, let $\phi^{\varepsilon}=\underline{w}-\varepsilon e^{r}-\varepsilon x^{2}$. We claim that $\phi^{\varepsilon} \leq w$ on $\mathbb{R} \times[0, t]$. Suppose not, then $w-\phi^{\varepsilon}$ can attain a global negative minimum, say, at $(\hat{x}, \hat{r})$. Since $w(x, 0)-\phi^{\varepsilon}(x, 0)=$ $w(x, 0)-\underline{w}(x, 0)+\varepsilon+\varepsilon x^{2} \geq \varepsilon, \hat{r}>0$. So that $\mathcal{L} \phi^{\varepsilon}(\hat{x}, \hat{r})=-\varepsilon e^{\hat{r}}+\varepsilon<0$. As a supersolution, $\max \left\{w(\hat{x}, \hat{r})-p(\hat{r}), \mathcal{L} \phi^{\varepsilon}(\hat{x}, \hat{r})\right\} \geq 0$, hence we must have $w(\hat{x}, \hat{r})-p(\hat{r}) \geq 0$. The condition $\underline{w} \leq p$ on $\mathbb{R} \times(0, t)$, and $p^{*}(t)=p(t)$ implies that $\underline{w} \leq p$ on $\mathbb{R} \times(0, t]$. Then $w(\hat{x}, \hat{r})<\phi^{\varepsilon}(\hat{x}, \hat{r})<\underline{w}(\hat{x}, \hat{r}) \leq p(\hat{r})$. This is a contradiction. Thus $\phi^{\varepsilon} \leq w$ in $\mathbb{R} \times(0, t]$ for each $\varepsilon>0$. Sending $\varepsilon \searrow 0$, we conclude that $\underline{w} \leq w$ in $\mathbb{R} \times(0, t]$.

In general, let $\left\{t_{n}\right\}$ be a sequence of positive numbers such that $t_{n} \nearrow t$ as $n \rightarrow \infty$, and $p(\cdot)$ is continuous at $t_{n}$. Then $\underline{w} \leq w$ in $\mathbb{R} \times\left[0, t_{n}\right]$. Sending $n \rightarrow \infty$ we obtain $\underline{w} \leq w$ in $\mathbb{R} \times[0, t)$.

As a subsolution, $w=\min \left\{p, w^{*}\right\}$. From the above argument, $w^{*}(s, t) \geq \underline{w}(s, t) \geq p(t)$. So that $w(s, t)=w_{*}(s, t)=p(t)$. By the definition of $b(\cdot)$, we conclude that $b(t) \geq s$.

Lemma 4.3 (A Criterion for Lower Bounds). For each $s<0<t$, let

$$
Q(s, r):=q(r)-\frac{2}{\sqrt{\pi}} \int_{-\infty}^{s / \sqrt{2 r}} e^{-z^{2}} d z
$$


Suppose $(s, t)$ is such that

$$
s<0<t, \quad Q(s, r) \leq Q(s, t) \quad \forall r \in(0, t) .
$$

Then $b(t) \geq s$.

Proof. Let $\underline{w}$ be the solution to

$$
\begin{cases}\mathcal{L} \underline{w}=0 & \text { in } \mathbb{R} \times(0, t] \\ \underline{w}(\cdot, 0)=\theta \mathbf{1}_{(2 s, 0)} & \text { on } \mathbb{R} \times\{0\} .\end{cases}
$$

where $\theta=p(t)\left(\frac{2}{\sqrt{\pi}} \int_{s / \sqrt{2 t}}^{0} e^{-z^{2}} d z\right)^{-1}$. We claim $\underline{w}$ satisfies (4.1).

1. Since the problem for $\underline{w}$ is linear, it can be expressed as:

$$
\underline{w}(x, r)=\theta \int_{2 s}^{0} \Gamma(x-y, r) d y=\frac{\theta}{\sqrt{\pi}} \int_{\frac{x}{\sqrt{2 r}}}^{\frac{x-2 s}{\sqrt{2 r}}} e^{-z^{2}} d z \quad \forall x \in \mathbb{R}, r>0 .
$$

In particular, when $x=s$,

$$
\underline{w}(s, t)=\frac{\theta}{\sqrt{\pi}} \int_{s / \sqrt{2 t}}^{-s / \sqrt{2 t}} e^{-z^{2}} d z=\frac{2 \theta}{\sqrt{\pi}} \int_{s / \sqrt{2 t}}^{0} e^{-z^{2}} d z=p(t) .
$$

2. By (4.2), we find

$$
\max _{x \in \mathbb{R}} \underline{w}(x, r)=\underline{w}(s, r)=\frac{p(t) \int_{s / \sqrt{2 r}}^{0} e^{-z^{2}} d z}{\int_{s / \sqrt{2 t}}^{0} e^{-z^{2}} d z} \quad \forall r>0 .
$$

For any $s<0<r$, we can compute

$$
\frac{2}{\sqrt{\pi}} \int_{s / \sqrt{2 r}}^{0} e^{-z^{2}} d z=1-\frac{2}{\sqrt{\pi}} \int_{-\infty}^{s / \sqrt{2 r}} e^{-z^{2}} d z=1+Q(s, r)-q(r)=Q(s, r)+p(r) .
$$

From (4.3) and (4.4), for all $r \in(0, t)$,

$$
\begin{aligned}
\max _{x \in \mathbb{R}} \underline{w}(x, r)-p(r) & =\frac{p(t)[Q(s, r)+p(r)]}{Q(s, t)+p(t)}-p(r) \\
& =\frac{p(t) Q(s, r)-p(r) Q(s, t)}{Q(s, t)+p(t)} \\
& \leq \frac{p(t)[Q(s, r)-Q(s, t)]}{Q(s, t)+p(t)} \leq 0,
\end{aligned}
$$

i.e., $\underline{w} \leq p$ on $\mathbb{R} \times(0, t)$. 
3. Since $Q(s, \cdot)$ is increasing for any $s<0, Q(s, t) \geq \lim _{r \backslash 0} Q(s, r)=0$. In particular when $r=t$, (4.4) reads as

$$
\frac{2}{\sqrt{\pi}} \int_{s / \sqrt{2 t}}^{0} e^{-z^{2}} d z=Q(s, t)+p(t) \geq p(t)
$$

So that $\theta \leq 1$ and thus $\underline{w}(\cdot, 0) \leq w(\cdot, 0)$ on $\mathbb{R} \times\{0\}$. Lemma 4.2 now gives $b(t) \geq s$.

Before we continue, we provide an interesting application of Lemmas 4.3 and 4.1.

Corollary 4.4. For each $t>0$, let $\zeta(t) \in(-\infty, 0)$ and $\nu(t) \in \mathbb{R}$ be defined by

$$
q(t)=\frac{2}{\sqrt{\pi}} \int_{-\infty}^{\zeta(t) / \sqrt{2 t}} e^{-z^{2}} d z=\frac{1}{\sqrt{\pi}} \int_{-\infty}^{\nu(t) / \sqrt{2 t}} e^{-z^{2}} d z
$$

1. Suppose $\zeta$ is a constant function. Then the exact solution to (1.1) is given by

$$
\begin{cases}w(x, t)=\frac{1}{\sqrt{\pi}} \int_{x / \sqrt{2 t}}^{(x-2 \zeta) / \sqrt{2 t}} e^{-z^{2}} d z & \forall x \geq \zeta, t>0, \\ b(t)=\zeta & \forall t>0 .\end{cases}
$$

2. Suppose $\zeta(r) \leq \zeta(t)$ for all $r \in(0, t)$. Then $\zeta(t) \leq b(t) \leq \nu(t)$.

3. Suppose $\dot{\zeta}(t) \geq 0$ for all $t \in(0, T]$. Then

$$
\zeta(t) \leq b(t) \leq \nu(t) \quad \forall t \in(0, T], \quad \lim _{t \searrow 0} \frac{b(t)}{\zeta(t)}=1 .
$$

Proof. 1. The first assertion may be verified by a direct computation. We note that it agrees with the formula for the first hitting time of Brownian motion to the level $\zeta$ (see e.g. [9] pages 94-96).

2. Suppose $\zeta(r) \leq \zeta(t) \forall r \in(0, t)$. Set $s=\zeta(t)$. Then

$$
Q(s, r)=q(r)-\frac{2}{\sqrt{\pi}} \int_{-\infty}^{\zeta(t) / \sqrt{2 r}} e^{-z^{2}} d z=\frac{2}{\sqrt{\pi}} \int_{\zeta(t) / \sqrt{2 r}}^{\zeta(r) / \sqrt{2 r}} e^{-z^{2}} d z \leq 0=Q(s, t)
$$

for each $r \in(0, t)$. Thus by Lemma $4.3, b(t) \geq s=\zeta(t)$. This is the lower bound for $b(t)$.

For $t>0$, Lemma 4.1 reads as

$$
\frac{1}{\sqrt{\pi}} \int_{-\infty}^{b(t) / \sqrt{2 t}} e^{-z^{2}} d z \leq q^{*}(t)=\frac{1}{\sqrt{\pi}} \int_{-\infty}^{\nu^{*}(t) / \sqrt{2 t}} e^{-z^{2}} d z,
$$

which implies that $b(t) \leq \nu^{*}(t)$. 
3. Suppose $\dot{\zeta}(t) \geq 0$ in $(0, T]$. Then by $(2) \zeta(t) \leq b(t) \leq \nu(t)$ for all $t \in(0, T]$. To complete the proof, it remains to estimate the difference between $\alpha(t):=\nu^{*}(t) / \sqrt{2 t}$ and $\gamma(t):=\zeta(t) / \sqrt{2 t}$. Let $\delta(t)=\ln 2 /(-2 \gamma(t)-1)$. Since $\lim _{t \backslash 0} \gamma(t)=-\infty$, we conclude that for all small positive $t>0, \delta(t) \in(0,1)$. Note that

$$
\begin{aligned}
\frac{1}{\sqrt{\pi}} \int_{-\infty}^{\alpha} e^{-z^{2}} d z & =q(t)=\frac{2}{\sqrt{\pi}} \int_{-\infty}^{\gamma} e^{-z^{2}} d z=\frac{2}{\sqrt{\pi}} \int_{-\infty}^{\gamma+\delta} e^{-z^{2}+2 \delta z-\delta^{2}} d z \\
& \leq \frac{2}{\sqrt{\pi}} \int_{-\infty}^{\gamma+\delta} e^{-z^{2}+2 \delta(\gamma+\delta)-\delta^{2}} d z=\frac{2 e^{\delta(2 \gamma+\delta)}}{\sqrt{\pi}} \int_{-\infty}^{\gamma+\delta} e^{-z^{2}} d z \\
& =\frac{2 e^{-\ln 2+\delta^{2}-\delta}}{\sqrt{\pi}} \int_{-\infty}^{\gamma+\delta} e^{-z^{2}} d z \leq \frac{1}{\sqrt{\pi}} \int_{-\infty}^{\gamma+\delta} e^{-z^{2}} d z
\end{aligned}
$$

Thus, $\alpha(t) \leq \gamma(t)+\delta(t)$. Then

$$
0 \leq 1-\frac{b(t)}{\zeta(t)} \leq 1-\frac{\nu(t)}{\zeta(t)}=1-\frac{\alpha(t)}{\gamma(t)} \leq \frac{\delta(t)}{-\gamma(t)}=\frac{\ln 2}{2 \gamma^{2}(t)+\gamma(t)}
$$

The third assertion of the Lemma thus follows by sending $t \rightarrow 0$.

Next we present a sufficient condition for $Q(s, \cdot)$ to attain its maximum in $(0, t]$ at $t$. Note that for $0<r<t, Q(s, r) \leq Q(s, t)$ is equivalent to

$$
\frac{q(t)-q(r)}{t-r} \geq \frac{2}{\sqrt{\pi}(t-r)}\left\{\int_{-\infty}^{s / \sqrt{2 t}} e^{-z^{2}} d z-\int_{-\infty}^{s / \sqrt{2 r}} e^{-z^{2}} d z\right\}=\frac{-s e^{-s^{2} /(2 \theta)}}{\theta^{3 / 2} \sqrt{2 \pi}}
$$

where $r<\theta \leq t$. The second equality follows from the mean value theorem. To achieve the maximum in $(0, t]$ for $Q(s, \cdot)$, it is sufficient to have

$$
\inf _{0<r<t} \frac{q(t)-q(r)}{t-r} \geq \sup _{r<\theta \leq t} \frac{|s| e^{-s^{2} /(2 \theta)}}{\theta^{3 / 2} \sqrt{2 \pi}}=\frac{|s| e^{-s^{2} /(2 t)}}{t^{3 / 2} \sqrt{2 \pi}}
$$

provided that $s \leq-\sqrt{3 t}$. Taking the best possible $s \leq-\sqrt{3 t}$ for the inequality (4.6) to hold, we then obtain the following.

Lemma 4.5. Assume that $t>0$ and

$$
k(t):=\inf _{0 \leq r \leq t} \frac{q(t)-q(r)}{t-r}>0 .
$$

Then

$$
b(t) \geq \max \left\{s \mid s \leq-\sqrt{3 t} ; \frac{|s|}{\sqrt{2 \pi} t^{3 / 2}} e^{-s^{2} /(2 t)} \leq k(t)\right\} .
$$

As an immediate consequence of the Lemma, we have 
Corollary 4.6. If $\dot{q}(t)>0$, then $b(t)>-\infty$.

We end this section with the following

Theorem 5. Assume that

$$
\limsup _{t \searrow 0} \frac{q(t)}{t \dot{q}(t)}<\infty
$$

Then

$$
\lim _{t \searrow 0} \frac{b(t)}{\sqrt{-2 t \log q(t)}}=-1 .
$$

Consequently, in special cases the following holds:

1. when $q(t)=A t^{m}$, where $A$ and $m$ are positive constants,

$$
b(t)=-\sqrt{-2 m t \log t}[1+o(1)], \quad \lim _{t \searrow 0} o(1)=0 ;
$$

2. when $q(t)=A e^{-\gamma^{2} /\left(2 t^{m}\right)}$, where $A, m, \gamma$ are positive constants,

$$
b(t)=-\gamma t^{(1-m) / 2}[1+o(1)], \quad \lim _{t \searrow 0} o(1)=0 .
$$

In particular,

$$
\lim _{t \searrow 0} b(t)= \begin{cases}-\infty & \text { if } m>1, \\ \gamma & \text { if } m=1, \\ 0 & \text { if } 0<m<1 .\end{cases}
$$

Proof. The idea is to estimate $k(r)$ via $q(r) / r$. Under the assumption (4.7), there exist positive constants $C$ and $T$ such that

$$
0<q(r) \leq \operatorname{Cr} \dot{q}(r) \quad \forall r \in(0, T]
$$

For any $0<r<t \leq T$, we can compute,

$$
\begin{aligned}
C(q(t)-q(r)) & \geq \int_{r}^{t} C \dot{q}(\theta) d \theta \geq \int_{r}^{t} \frac{q(\theta)}{\theta} d(\theta-r) \\
& =\left.\frac{q(\theta)(\theta-r)}{\theta}\right|_{\theta=r} ^{\theta=t}-\int_{r}^{t}(\theta-r) \frac{\theta \dot{q}(\theta)-q(\theta)}{\theta^{2}} d \theta \\
& =\frac{(t-r) q(t)}{t}-\int_{r}^{t} \dot{q}(\theta) d \theta+\int_{r}^{t} \frac{r \theta \dot{q}(\theta)+(\theta-r) q(\theta)}{\theta^{2}} d \theta \\
& \geq(t-r) \frac{q(t)}{t}-[q(t)-q(r)] .
\end{aligned}
$$


That is, $(C+1)(q(t)-q(r)) \geq(t-r) q(t) / t$. It follows that

$$
k(t)=\inf _{0<r<t} \frac{q(t)-q(r)}{t-r} \geq \frac{1}{C+1} \frac{q(t)}{t} .
$$

Now fix $t \in(0, T]$, when $s \leq-\sqrt{3 t}$,

$$
\frac{|s|}{t^{3 / 2} \sqrt{2 \pi}} \int_{-\infty}^{s / \sqrt{2 t}} e^{-z^{2}} d z \leq \frac{|s|}{t^{3 / 2} \sqrt{2 \pi}} e^{s^{2} /(2 t)} .
$$

Let $s<-\sqrt{3 t}$ be the solution to

$$
\frac{|s|}{\sqrt{2 \pi} t^{3 / 2}} \int_{-\infty}^{s / \sqrt{2 t}} e^{-z^{2}} d z=\frac{1}{C+1} \frac{q(t)}{t} .
$$

For small $t, q(t)$ is small, so that $s / \sqrt{t} \ll-1$ and we can use the expansion, for $a<0$,

$$
\int_{-\infty}^{a} e^{-z^{2}} d z=\int_{a^{2}}^{\infty} \frac{e^{-x} d x}{2 \sqrt{x}}=\frac{e^{-a^{2}}}{2|a|}-\int_{a^{2}}^{\infty} \frac{e^{-x} d x}{4 x^{3 / 2}}=\frac{e^{-a^{2}}}{2|a|}\left\{1-\frac{\theta}{2 a^{2}}\right\}
$$

where $\theta=\theta(a) \in(0,1)$. Hence, the equation for $s$ reads

$$
e^{-s^{2} /(2 t)}\left\{1-\frac{\theta}{s^{2} /(2 t)}\right\}=\frac{\sqrt{2 \pi}}{C+1} q(t) .
$$

It then follows that

$$
\begin{aligned}
|s| & =\sqrt{2 t}\left(-\log q(t)+\log \left(1-\theta t / s^{2}\right)+\log [(C+1) / \sqrt{2 \pi}]\right)^{1 / 2} \\
& \leq \sqrt{-2 t \log q(t)}\left\{1+\frac{\log [(C+1) / \sqrt{4 \pi}]}{-\log q(t)}\right\}^{1 / 2} .
\end{aligned}
$$

By Lemma 4.5, we then have

$$
\begin{aligned}
b(t) & \geq s \geq-\sqrt{-2 t \log q(t)}\left\{1+\frac{\log [(C+1) / \sqrt{2 \pi}]}{|\log q(t)|}\right\}^{1 / 2} \\
& =-\sqrt{-2 t \log q(t)}\{1+o(1)\} .
\end{aligned}
$$

This gives the lower bound for $b(t)$, now we estimate the upper bound. From Lemma $(4.1)$,

$$
q(t) \geq \frac{1}{\sqrt{\pi}} \int_{-\infty}^{b(t) / \sqrt{2 t}} e^{-z^{2}} d z=\frac{\sqrt{t}}{\sqrt{2 \pi}|b(t)|}\left\{1-\frac{\theta t}{b^{2}(t)}\right\} e^{-b(t)^{2} /(2 t)} .
$$

This implies that

$$
\begin{aligned}
b(t) & \leq-\sqrt{-2 t \log q(t)}\left\{1+\frac{\log \left[1-\theta t / b^{2}(t)\right]-\log [\sqrt{2 \pi}|b(t)| / \sqrt{t}]}{-\log q(t)}\right\}^{1 / 2} \\
& \leq-\sqrt{-2 t \log q(t)}\left\{1-\frac{O(1) \log |\log q(t)|}{|\log q(t)+o(1)|}\right\}^{1 / 2} .
\end{aligned}
$$

The assertion (4.8) thus follows. The remainder of the theorem is a direct application of (4.8). 


\section{$5 \quad$ Integral Equations}

As in $\S 4$, we assume $\sigma \equiv 1$ and $\mu \equiv 0$. Also we assume that $p$ (and therefore $q$ ) is continuous. Then the solution to (3.12) can be expressed as

$$
\begin{aligned}
w(x, t) & =\int_{-\infty}^{0} \Gamma(x-y, t) d y+\int_{0}^{t} d p(s) \int_{-\infty}^{b(s)} \Gamma(x-y, t-s) d y \\
& =1-\int_{0}^{\infty} \Gamma(x-y, t) d y+\int_{0}^{t} d p(s)\left(1-\int_{b(s)}^{\infty} \Gamma(x-y, t-s) d y\right) \\
& =p(t)-\int_{-\infty}^{x} \Gamma(z, t) d z+\int_{0}^{t} d q(s) \int_{-\infty}^{x-b(s)} \Gamma(z, t-s) d z,
\end{aligned}
$$

where the second equation is obtained by using $\int_{\mathbb{R}} \Gamma(x-y, s) d y=1 \forall s>0$.

Now assume that $b$ is smooth. Differentiate $w$, we can derive

$$
u(x, t)=-w_{x}(x, t)=\Gamma(x, t)-\int_{0}^{t} \Gamma(x-b(s), t-s) d q(s) .
$$

Also, for $x \neq b(t)$, we can further differentiate to obtain

$$
\begin{gathered}
u_{x}(x, t)=\Gamma_{x}(x, t)-\int_{0}^{t} \Gamma_{x}(x-b(s), t-s) d q(s) \\
u_{t}(x, t)=\Gamma_{t}(x, t)-\int_{0}^{t} \Gamma_{t}(x-b(s), t-s) d q(s) \\
=\Gamma_{t}(x, t)+\int_{0}^{t}\left(\frac{d}{d s} \Gamma(x-b(s), t-s)+\dot{b}(s) \Gamma_{x}(x-b(s), t-s)\right) d q(s) \\
=\Gamma_{t}(x, t)+\int_{0}^{t} \dot{b}(s) \Gamma_{x}(x-b(s), t-s) d q(s)+\int_{0}^{t} \dot{q}(s) d \Gamma(x-b(s), t-s) \\
=\Gamma_{t}(x, t)+\int_{0}^{t} \dot{b}(s) \Gamma_{x}(x-b(s), t-s) d q(s)-\dot{q}(0) \Gamma(x, t) \\
-\int_{0}^{t} \Gamma(x-b(s), t-s) d \dot{q}(s),
\end{gathered}
$$

where the second equation is obtained by the equality

$$
\Gamma_{t}(x-b(s), t-s)=-\frac{d}{d s} \Gamma(x-b(s), t-s)-\dot{b}(s) \Gamma_{x}(x-b(s), t-s),
$$

and the third equation by using integration by parts to $\int_{0}^{t} \dot{q}(s) d \Gamma(x-b(s), t-s)$.

From potential theory, for any $b$ and $f$ with the certain regularity, we have

$$
\lim _{x \rightarrow b(t) \pm 0} \int_{0}^{t} f(s) \Gamma_{x}(x-b(s), t-s) d s=\int_{0}^{t} f(s) \Gamma_{x}(b(t)-b(s), t-s) d s \mp f(t) .
$$


It can be derived as follow.

$$
\begin{aligned}
& \lim _{x \rightarrow b(t) \pm 0} \int_{0}^{t} f(s) \Gamma_{x}(x-b(s), t-s) d s \\
= & -\lim _{x \rightarrow b(t) \pm 0} \int_{0}^{t} f(s)\left[\frac{(b(t)-b(s))}{\sqrt{2 \pi}(t-s)^{3 / 2}} e^{-\frac{(x-b(s))^{2}}{2(t-s)}}+\frac{(x-b(t))}{\sqrt{2 \pi}(t-s)^{3 / 2}} e^{-\frac{(x-b(s))^{2}}{2(t-s)}}\right] d s \\
= & \int_{0}^{t} f(s) \Gamma_{x}(b(t)-b(s), t-s) d s-\lim _{x \rightarrow b(t) \pm 0} \int_{0}^{t} f(s) \frac{x-b(t)}{\sqrt{2 \pi}(t-s)^{3 / 2}} e^{-\frac{(x-b(s))^{2}}{2(t-s)}} d s \\
= & \int_{0}^{t} f(s) \Gamma_{x}(b(t)-b(s), t-s) d s-\frac{2}{\sqrt{\pi}} \lim _{x \rightarrow b(t) \pm 0} \int_{\frac{x-b(t)}{\sqrt{2 t}}}^{ \pm \infty} f\left(t-\frac{(x-b(t))^{2}}{2 \eta^{2}}\right) e^{-\left(\eta+\frac{b(t)-b(s)}{\sqrt{2(t-s)})^{2}}\right.} d \eta \\
= & \int_{0}^{t} f(s) \Gamma_{x}(b(t)-b(s), t-s) d s-\frac{2}{\sqrt{\pi}} \int_{0}^{ \pm \infty} f(t) e^{-\eta^{2}} d \eta \\
= & \int_{0}^{t} f(s) \Gamma_{x}(b(t)-b(s), t-s) d s \mp f(t) .
\end{aligned}
$$

Note $w(x, t)=p$ and $0=u(x, t)=u_{x}(x, t)=u_{t}(x, t)$ for $x<b(t)$. Sending $x$ to $b(t)$ from below in (5.1) and (5.2) we then obtain

$$
\begin{aligned}
\int_{-\infty}^{b(t)} \Gamma(z, t) d z & =\int_{0}^{t} d q(s) \int_{-\infty}^{b(t)-b(s)} \Gamma(z, t-s) d z \\
\Gamma(b(t), t) & =\int_{0}^{t} \Gamma(b(t)-b(s), t-s) d q(s),
\end{aligned}
$$

which reflect the free boundary condition $w(b(t), t)=p(t)$ and the condition $u(b(t), t)=0$ respectively. Sending $x$ to $b(t)$ from below in (5.3) and (5.4) and use (5.5), we have

$$
\begin{aligned}
\dot{q}(t)= & \Gamma_{x}(b(t), t)-\int_{0}^{t} \Gamma_{x}(b(t)-b(s), t-s) d q(s) \\
-\dot{b}(t) \dot{q}(t)= & \Gamma_{t}(b(t), t)+\int_{0}^{t} \dot{b}(s) \Gamma_{x}(b(t)-b(s), t-s) d q(s) \\
& -\dot{q}(0) \Gamma(b(t), t)-\int_{0}^{t} \Gamma(b(t)-b(s), t-s) d \dot{q}(s) .
\end{aligned}
$$

(5.8) reflect the free boundary condition $u_{x}\left(b(t)^{-}, t\right)=0$ and $u_{x}\left(b(t)^{+}, t\right)=\dot{q}(t)$. Similarly, (5.9) reflects the free boundary condition that $u_{t}\left(b(t)^{-}, t\right)=0$ and $u_{t}\left(b(t)^{+}, t\right)=$ $-\dot{b}(t) u_{x}\left(b(t)^{+}, t\right)=-\dot{b}(t) \dot{q}(t)$.

Clearly, these identities can provide numerical schemes much more flexible and economic than integrating the corresponding PDEs. For this purpose, it is necessary to study solutions to each of these identities. 
One observes that if $b(\cdot)$ is a solution to $(5.7)$, then $b_{1}(t):=-b(t)$ is also the solution as well. Hence, we need to be careful when considering solutions to the integral equation.

Theorem 6. Let $q:[0, \infty) \rightarrow[0,1)$ be continuous, increasing, and $q(0)=0$. Assume that $b:(0, T] \rightarrow \mathbb{R}$ is a continuous function. Then $x=b(t), t \in(0, T]$, is the solution to the free boundary problem provided that one of the following holds.

1. b satisfies (5.6) for all $t \in(0, T]$;

2. $b$ satisfies (5.7) for all $t \in(0, T], b(t)<0$ for all sufficiently small positive $t$, and the function

$$
t \rightarrow q_{1 / 2}(t):=\int_{0}^{t} \frac{\dot{q}(t)}{\sqrt{2 \pi(t-s)}} d s
$$

is continuous in $(0, T]$ with $q_{1 / 2}(0+)=0$;

3. $b$ satisfies (5.8), $\lim _{t \backslash 0} \frac{b(t)}{\sqrt{t}}=-\infty, \dot{q}$ is continuous in $[0, T]$, and the function

$$
t \rightarrow q_{3 / 2}^{b}:=\int_{0}^{t} \frac{|b(t)-b(s)|}{\sqrt{2 \pi}(t-s)^{3 / 2}} d q(s)
$$

is continuous on $(0, T]$ and is uniformly bounded.

The analogous condition for (5.9) is too technical and hence we omit it here.

Proof. With the given continuous function $b$, we define $w(x, t)$ as in (5.1). Note that

$$
\begin{aligned}
0 & \geq w(x, t)-w_{0}(x, t)=\int_{0}^{t} d p(s) \int_{-\infty}^{b(s)} \Gamma(x-y, t-s) d y \\
& =\int_{0}^{t} d p(s)\left(1-\int_{b(s)}^{\infty} \Gamma(x-y, t-s) d y\right) \\
& =-q(t)+\int_{0}^{t} d q(t) \int_{-\infty}^{x-b(s)} \Gamma(z, t-s) d z \geq-q(t) .
\end{aligned}
$$

This implies that

$$
\left|w(x, t)-w_{0}(x, t)\right| \leq q(t) .
$$

When $t=0$, it reads $\left|w(x, 0)-w_{0}(x, 0)\right| \leq q(0)=0$, so that $w(x, 0)=w_{0}(x, 0)=\mathbf{1}_{(-\infty, 0)}$. In addition, upon differentiation, $\mathcal{L} w=\dot{p} \mathbf{1}_{\{x<b(t)\}} \leq 0$ as a measure in $\mathbb{R} \times(0, T]$. This can verified as follows. Direct calculation gives

$$
\begin{aligned}
w_{x x} & =-\Gamma_{x}(x, t)+\int_{0}^{t} \Gamma_{x}(x-b(s), t-\tau) d q(s), \\
w_{t} & =\dot{p}(t)-\int_{-\infty}^{x} \Gamma_{t}(z, t) d z+\int_{0}^{t} \int_{-\infty}^{x-b(s)} \Gamma_{t}(z, t-s) d z d q(s) .
\end{aligned}
$$


Using the fact that $\Gamma_{t}=\frac{1}{2} \Gamma_{x x}$, we obtain

$$
\int_{-\infty}^{x} \Gamma_{t}(z, t) d z=\int_{-\infty}^{x} \frac{1}{2} \Gamma_{x x}(z, t) d z=\left.\frac{1}{2} \Gamma_{x}(z, t)\right|_{z=-\infty} ^{z=x}=\frac{1}{2} \Gamma_{x}(x, t) .
$$

When $x<b(s)$,

$$
\begin{aligned}
\int_{0}^{t} \int_{-\infty}^{x-b(s)} \Gamma_{t}(z, t-s) d z d q(s) & =\left.\frac{1}{2} \int_{0}^{t} \Gamma_{x}(z, t-s)\right|_{z=-\infty} ^{z=x-b(s)} d q(s) \\
& =\frac{1}{2} \int_{0}^{t} \Gamma_{x}(x-b(s), t-\tau) d q(s) .
\end{aligned}
$$

When $x>b(s)$,

$$
\begin{aligned}
& \int_{0}^{t} \int_{-\infty}^{x-b(s)} \Gamma_{t}(z, t-s) d z d q(s) \\
= & \left.\frac{1}{2} \int_{0}^{t} \Gamma_{x}(z, t-s)\right|_{z=-\infty} ^{z=(b(t)-b(s))^{-}} d q(s)+\left.\frac{1}{2} \int_{0}^{t} \Gamma_{x}(z, t-s)\right|_{z=(b(t)-b(s))^{+}} ^{z=x-b(s)} d q(s) \\
= & \frac{1}{2} \int_{0}^{t} \Gamma_{x}(x-b(s), t-s) d q(s)+\lim _{z \nearrow(b(t)-b(s))} \frac{1}{2} \int_{0}^{t} \Gamma_{x}(z, t-s) d q(s) \\
& -\lim _{z \searrow(b(t)-b(s))} \frac{1}{2} \int_{0}^{t} \Gamma_{x}(z, t-s) d q(s) . \\
= & \dot{q}(t)+\frac{1}{2} \int_{0}^{t} \Gamma_{x}(x-b(s), t-s) d q(s) . \\
= & -\dot{p}(t)+\frac{1}{2} \int_{0}^{t} \Gamma_{x}(x-b(s), t-s) d q(s) .
\end{aligned}
$$

Thus $\mathcal{L} w=\dot{p} \mathbf{1}_{\{x<b(t)\}} \leq 0$ in $\mathbb{R} \times(0, T]$ holds.

It remains to show that $w(x, t)=p(t)$ for $x \leq b(t)$ and $w<p(t)$ for $x>b(t)$.

(1) Assume the condition of the first assertion. We define

$$
v(x, t):=w(x, t)-p(t)=-\int_{-\infty}^{x} \Gamma(z, t) d z+\int_{0}^{t} d q(s) \int_{-\infty}^{x-b(s)} \Gamma(z, t-s) d z .
$$

Upon differentiation, $\mathcal{L} v=0$ in $\{x<b(t)\}$. Note that $v$ is bounded, continuous and, by (5.6), $v(b(t), t)=0$. It follows that $v(x, t) \equiv 0$ for all $x \leq b(t)$, i.e., $w=p(t)$ for any $x \leq b(t)$. Also by differentiation, we see that $\mathcal{L} v=-\dot{p} \leq 0$ in $\{x>b(t)\}$. The strong maximum principle gives $v<0$ in $\{x>b(t)\}$. That is $w<p(t)$ in $\{x>b(t)\}$. Thus $w$ is a variational solution.

(2) Assume the condition of the second assertion. We see that $u:=-w_{x}$ given by (5.2) is continuous in $\mathbb{R} \times(0, \infty)$. For every small $\varepsilon>0$, the function $u$ satisfies $\mathcal{L} u=0$ in

$$
\Omega_{\varepsilon}:=\{(x, t) \mid x<b(t), t \in(\varepsilon, T]\} .
$$


Also, from equation $(5.7), u(b(t), t)=0$ for all $t \in(0, T]$. Since $b(t)<0$ for small positive $t$, we can assume that $b(\varepsilon)<0$. It then follows from (5.2) that for all $x \leq b(\varepsilon)$,

$$
|u(x, \varepsilon)| \leq \max \left\{\Gamma(x, \varepsilon), q_{1 / 2}(\varepsilon)\right\} \leq \max \left\{\Gamma(b(\varepsilon), \varepsilon), q_{1 / 2}(\varepsilon)\right\} \leq q_{1 / 2}(\varepsilon),
$$

since for any $t \in(0, T]$

$$
\int_{0}^{t} \Gamma(b(t)-b(s), t-s) d q(s) \leq \int_{0}^{t} \frac{\dot{q}(s)}{\sqrt{2 \pi(t-s)}} d s=q_{1 / 2}(t),
$$

which holds for $\varepsilon$ as well. It then follows from the maximum principle that

$$
\max _{\Omega_{\varepsilon}}|u| \leq q_{1 / 2}(\varepsilon)
$$

Sending $\varepsilon$ to 0 from above, we obtain $w_{x}=u \equiv 0$ in $\{(x, t) \mid x \leq b(t), t>0\}$. $w$ is constant in $\{x \leq b(t)\}$ and $w(-\infty, t)=p(t)$ imply that $w \equiv p(t)$ in $\{x \leq b(t)\}$. From the first assertion, the second assertion of the Theorem thus holds.

(3) Assume the conditions in the third assertion. Let $u:=-w_{x}$ be given by (5.2) and $u_{x}:=-w_{x x}$ by (5.3) when $x \neq b(t)$. Since $b, \dot{q}$, and $q_{3 / 2}^{b}$ are continuous, and (5.5) holds for $f=\dot{q}$, sending $x$ to $b(t)$ from below in the equation for $u_{x}$ and using (5.8) we derive that $u_{x}\left(b(t)^{-}, t\right)=0$.

Next we show that $u_{x} \equiv 0$ in $\{x<b(t)\}$. To do this, we first show that that $u_{x}$ given in (5.3) is uniformly bounded in $\{x<b(t)\}$. First of all, the boundedness of $q_{3 / 2}^{b}$ and (5.8) implies that $\Gamma_{x}(b(t), t)$ is uniformly bounded in $(0, T]$. Next, as $b(t)<-\sqrt{3 t}$ for small positive $t$, we see that $0<\Gamma_{x}(x, t)<\Gamma(b(t), t)$ for all $x<b(t)$. Thus $\Gamma_{x}(x, t)$ is bounded for all $x<b(t)$.

For $x<b(t)$, let $A_{1}=\{s \in(0, t]|b(t)-x>2| b(t)-b(s) \mid\}$ and $A_{2}=[0, t] \backslash A_{1}$. Then

$$
\int_{0}^{t} \Gamma_{x}(x-b(t), t-s) d q(s)=I_{1}+I_{2}, \quad I_{i}=\int_{A_{i}} \Gamma_{x}(x-b(t), t-s) d q(s) .
$$

Note that

$$
\left|I_{2}\right| \leq \int_{0}^{t} \frac{|b(t)-b(s)| \dot{q}(s)}{2 \sqrt{\pi}|t-s|^{3 / 2}} d s \leq 2 q_{3 / 2}^{b}(t)
$$

is uniformly bounded. To estimate $I_{i}$, notice that when $x-b(t)>2|b(t)-b(s)|,(x-$ $b(s))^{2}=\left(x-b(t)-(b(t)-b(s))^{2} \geq \frac{1}{4}(x-b(t))^{2}\right.$. Thus,

$$
\left|I_{1}\right| \leq \int_{0}^{t} \frac{|x-b(t)| \dot{q}(s) e^{-|x-b(t)|^{2} /[16(t-s)]} d s}{\sqrt{2 \pi}(t-s)^{3 / 2}} \leq\|\dot{q}\|_{\infty} .
$$


and therefore $u_{x}$ is uniformly bounded in $\{x<b(t)\}$.

Since $\mathcal{L} u_{x}=0$ in $\left.\{x<b(t), t>0\}, u_{x}(b(t)-0), t\right)=0$, and $u_{x}(x, 0)=0$ for all $x<0$, a special maximum principle then implies that $u_{x} \equiv 0$ in $\{x<b(t)\}$. Using $u(-\infty, t)=0$ we then conclude that $u \equiv 0$. Following (2), the third assertion of the Theorem follows.

\section{References}

[1] M. Avellaneda And J. Zhu, Modeling the distance-to-default process of a firm, Risk, 14 (2001), pp. 125-129.

[2] T. Bielecki And M. Rutkowski, Credit Risk: Modelling, Valuation and Hedging, Springer, 2001.

[3] M. Crandall, H. Ishit, And P. Lions, User's guide to viscosity solutions of second order partial differential equations, Bulletin of the American Mathematical Society, 27 (1992), pp. 1-67.

[4] L. Evans, Partial Differential Equations, American Matheamtical Society, 1998.

[5] A. Friedman, Partial Differential Equations of Parabolic Type, Prentice-Hall, 1964.

[6] _ _ Variational Principles and Free-Boundary Problems, John Wiley \& Sons, 1982.

[7] J. Hull And A. White, Valuing credit default swaps ii: Modeling default correlations, Journal of Derivatives, 8 (2001), pp. 12-22.

[8] I. Iscoe And A. KReinin, Default boundary problem. Algorithmics Inc., Internal Paper., 2002.

[9] I. Karatzas And S. Shreve, Brownian Motion and Stochastic Calculus, Springer, 1996.

[10] B. O. KSEndal, Stochastic Differential Equations, Springer, sixth ed., 2002.

[11] R. Merton, On the pricing of corporate debt: The risk structure of interest rates, Journal of Finance, 29 (1974), pp. 449-470.

[12] G. PeskiR, Limit at zero of the Brownian first-passage density, Probability Theory and Related Fields, 124 (2002), pp. 100-111. 
[13] _ On integral equations arising in the first-passage problem for Brownian motion, Integral Equations Appl., 14 (2002), pp. 397-423.

[14] L. Ricciardi, A. Di Crescenzo, V. Giorno, and A. Nobile, An outline of theoretical and algorithmic approaches to first passage time problems with applications to biological modeling, Math. Japonica, 50 (1999), pp. 247-322.

[15] C. Zucca, L. Sacerdote, And G. Peskir, On the inverse first-passage problem for a Wiener process. Preprint. 